\title{
Radiation-Induced Oral Mucositis
}

\author{
Osama Muhammad Maria ${ }^{1,2,3}$, Nicoletta Eliopoulos ${ }^{3,4}$ and Thierry Muanza ${ }^{1,2,3,5 *}$ \\ ${ }^{1}$ Faculty of Medicine, Experimental Medicine Department, McGill University, Montreal, QC, Canada, ${ }^{2}$ Radiation Oncology \\ Department, Jewish General Hospital, McGill University, Montreal, QC, Canada, ${ }^{3}$ Lady Davis Institute for Medical Research, \\ Jewish General Hospital, McGill University, Montreal, QC, Canada, ${ }^{4}$ Faculty of Medicine, Surgery Department, McGill \\ University, Montreal, QC, Canada, ${ }^{5}$ Oncology Department, McGill University, Montreal, QC, Canada
}

Radiation-induced oral mucositis (RIOM) is a major dose-limiting toxicity in head and neck cancer patients. It is a normal tissue injury caused by radiation/radiotherapy (RT), which has marked adverse effects on patient quality of life and cancer therapy continuity. It is a challenge for radiation oncologists since it leads to cancer therapy interruption, poor local tumor control, and changes in dose fractionation. RIOM occurs in 100\% of altered fractionation radiotherapy head and neck cancer patients. In the United Sates, its economic cost was estimated to reach 17,000.00 USD per patient with head and neck cancers. This review will discuss RIOM definition, epidemiology, impact and side effects, pathogenesis, scoring scales, diagnosis, differential diagnosis, prevention, and treatment.

Keywords: chemotherapy, oral mucositis, radiation, radiotherapy, normal tissue injury, pathobiology, mesenchymal stromal/stem cells

\section{OPEN ACCESS}

Edited by: Issam El Naqa,

University of Michigan, USA

Reviewed by:

Michael Andrew Samuels,

Yale University, USA

John Staffurth,

Cardiff University, UK

${ }^{*}$ Correspondence:

Thierry Muanza

tmuanza@yahoo.com

Specialty section:

This article was submitted to Radiation Oncology, a section of the journal

Frontiers in Oncology

Received: 10 June 2016 Accepted: 21 April 2017

Published: 22 May 2017

Citation:

Maria OM, Eliopoulos N and Muanza T (2017) Radiation-

Induced Oral Mucositis.

Front. Oncol. 7:89.

doi: 10.3389/fonc.2017.00089

\section{DEFINITION}

Radiation-induced oral mucositis (RIOM) (Figures 1, 4 and 5C) is one of the major ionizing radiation toxicities and normal tissue injuries that result from radiotherapy (1). RIOM was first termed in 1980 as a side effect of radiotherapy (RT) in cancer patients (2). RIOM is a normal tissue injury lasting between 7 and 98 days, which starts as an acute inflammation of oral mucosa, tongue, and pharynx after RT exposure $(1,3)$. This coincides with recruitment of various inflammatory cells and release of inflammatory cytokines, chemotactic mediators, and growth factors. RIOM can progress to an acute life-threatening stage as a result of severe physical obstruction of food and water intake with subsequent weight loss and septic complication due to lost protective epithelial and basement membrane barriers. This leads to limitations of local tumor control due to cancer treatment interruption and alterations in radiation dose fractionation (4-7). Studies suggested the stages of progression of RIOM as initial hyperemia and erythema during the preulcer phase, during which there is a release of various pro-inflammatory cytokines from epithelial, vascular, and connective tissue cells at the site of tissue injury. This is followed by the epithelial phase with various degrees of desquamation and basement membrane damage with loss of the protective barrier, which ends with the physical appearance of the ulceration. The postulcerative phase varies depending on the extent of the tissue toxicity. A secondary infection with Gram-negative bacteria or yeast may occur, with microcoagulation of the vasculature that worsens the inflammation by the local ischemia with more necrotic tissue yield. The final stage will be the healing phase and fibrosis $(2,8,9)$.

\section{RIOM EPIDEMIOLOGY (INCIDENCE, PREDICTORS, AND RISK FACTORS)}

Radiation-induced oral mucositis occurs in up to $80 \%$ of head and neck cancer irradiated patients and reaches up to $100 \%$ in patients with altered fractionation head and neck cancer. RIOM of grade 3 and 4 have been recorded in $56 \%$ of head and neck cancer patients treated with radiotherapy $(1,12)$. 


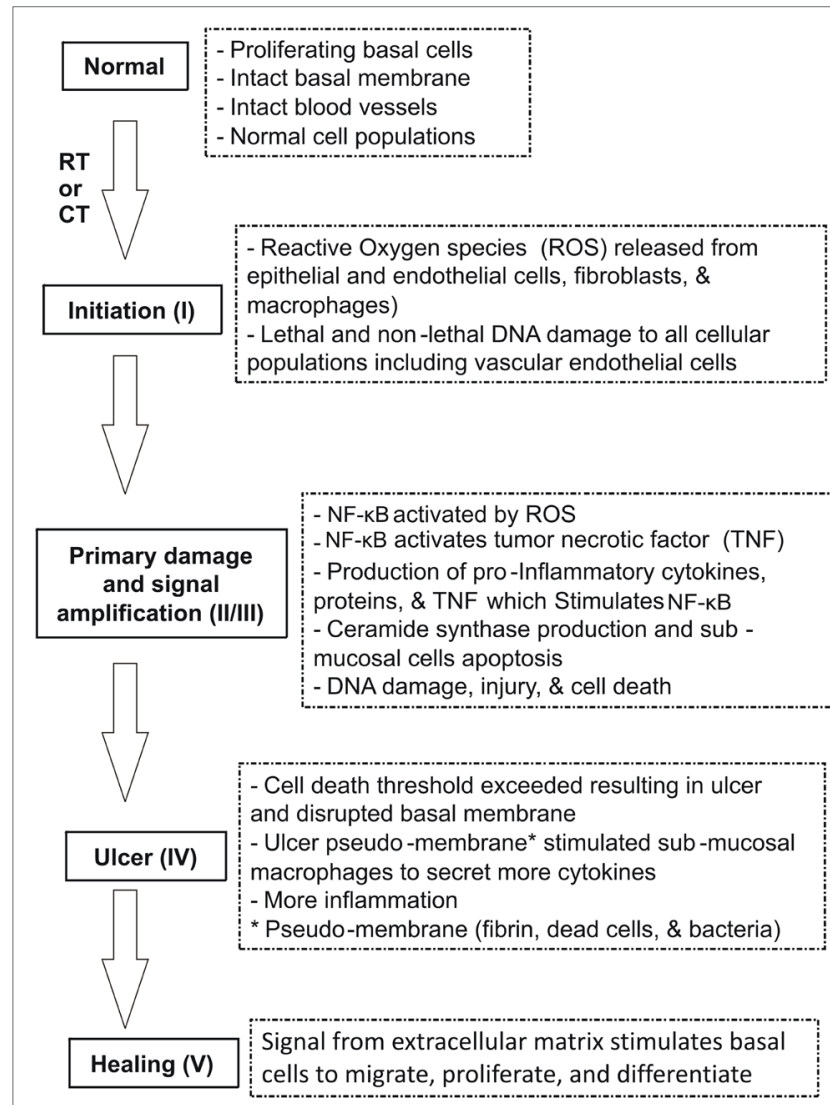

FIGURE 1 | Pathobiology of oral mucositis (OM) (10). Sonis has suggested five stages (phases) of OM injury induced by radiotherapy (RT) and/or chemotherapy (CT): initiation, signaling, amplification, ulceration, and healing. The pathogenesis of each phase is illustrated.

Many risk factors have been identified for RIOM. These risk factors include concomitant chemotherapy (CT), bad oral hygiene, below average nutritional stratus, lack of antibiotic use at early stage mucositis, and smoking (13).

Table 1 shows the significant predictors for the prevalence of severe RIOM and the symptoms of RIOM in a longitudinal study of patients with oral cavity cancer among head and neck cancer outpatients of a radiation department at a major medical center in Taiwan (14). This study used the Generalized Estimating Equations to analyze the predictive factors of prevalence of severe RIOM and RIOM-related symptoms. They found that the significant predictors for the prevalence of severe RIOM included type of treatment [RT vs. concomitant chemoradiotherapy (CCRT)], cumulative radiation dose, smoking, and body mass index (BMI). Patients who received CCRT (Coef. 0.145, $p<0.05$ ), who have a higher cumulative radiation dose (Coef. $0.000, p<0.01$ ), who are smokers (Coef. 0.090, $p<0.01$ ), and who have lower BMI (Coef. $0.005, p<0.05)$ were at high risk to develop severe RIOM. RIOMrelated symptoms were also predicted by type of treatment (RT vs. CCRT) (Coef. 1.618, $p<0.05$ ), cumulative radiation dose (Coef. $0.003, p<0.05$ ), and smoking (Coef. 1.759, $p<0.001$ ). These significant predictors are implemented by radiation oncologists to minimize and/or prevent the RIOM. June Eilers and Rita Million
TABLE 1 | Data analysis for RIOM predictors using IBM SPSS version 21.0 (Armonk, NY, USA) (14).

\begin{tabular}{lrrrrrr}
\hline Variable & Coef. & SE & z & $\boldsymbol{p}>\mathbf{z}$ & $\begin{array}{r}\text { 95\% conf. } \\
\text { interval }\end{array}$ \\
\hline Prevalence of severe RIOM & & & & & & \\
$\begin{array}{l}\text { Treatment type } \\
\text { (RT vs. CCRT) }\end{array}$ & 0.145 & 0.06 & 5.65 & 0.017 & 0.03 & 0.26 \\
Cumulative RT dose (cGy) & 0.000 & 0.03 & 16.47 & 0.001 & -0.00 & 0.01 \\
Smoking (no vs. yes) & 0.090 & 0.03 & 8.52 & 0.004 & 0.03 & 0.15 \\
Body mass index & -0.005 & 0.01 & 4.56 & 0.033 & -0.10 & 0.00 \\
Time & 0.417 & 0.09 & 23.56 & 0.001 & 0.25 & 0.59 \\
Intercept & -0.277 & 0.17 & 2.72 & 0.099 & -0.61 & 0.05 \\
Symptoms of RIOM & & & & & & \\
Treatment type (RT vs. & 1.618 & 0.49 & 10.76 & 0.001 & 0.65 & 2.59 \\
CCRT) & & & & & & \\
Cumulative RT dose (cGy) & 0.003 & 0.01 & 4.03 & 0.045 & -0.01 & -0.01 \\
Smoking (no vs. yes) & 1.759 & 0.41 & 18.50 & 0.000 & 0.96 & 2.56 \\
Body mass index & -0.002 & 0.05 & 0.00 & 0.973 & -0.09 & 0.09 \\
Time & 1.338 & 1.34 & 2.57 & 0.109 & -0.48 & 4.77 \\
Intercept & 6.023 & 2.77 & 4.74 & 0.030 & 0.60 & 11.45 \\
\hline
\end{tabular}

Significant predictors for RIOM.

Significant predictors for the prevalence of severe RIOM (CCRT, cumulative radiation dose, smoking, and low BMI) and the symptoms of RIOM in a longitudinal study of patients with oral cavity cancer among head and neck patients (CCRT, cumulative radiation dose, and smoking).

CCRT, concomitant chemoradiotherapy; RIOM, radiation-induced oral mucositis.

TABLE 2 | Patient-linked factors leading to increased risk for oral mucositis (OM) (15).

\begin{tabular}{|c|c|}
\hline Age & $\begin{array}{l}\text { Increased risk in very young age (high cell turnover } \\
\text { rate) and old age (slower healing rate) }\end{array}$ \\
\hline Gender & Trends to increased risk in females \\
\hline Oral health and hygiene & $\begin{array}{l}\text { Maintaining good oral hygiene and oral health lowers } \\
\text { radiation-induced oral mucositis (RIOM) risk }\end{array}$ \\
\hline $\begin{array}{l}\text { Salivary secretory } \\
\text { function }\end{array}$ & Decreased saliva leads to increased RIOM risk \\
\hline Genetic factors & $\begin{array}{l}\text { Potential for high RIOM risk in certain individuals still } \\
\text { to be identified }\end{array}$ \\
\hline Body mass index & $\begin{array}{l}\text { Delayed healing and increased breakdown in } \\
\text { malnourished individuals }\end{array}$ \\
\hline Renal function & $\begin{array}{l}\text { Increased mucotoxicity linked with high serum } \\
\text { creatinine level (poor renal function) }\end{array}$ \\
\hline Smoking & Delays the healing \\
\hline $\begin{array}{l}\text { Previous cancer } \\
\text { treatment }\end{array}$ & $\begin{array}{l}\text { History of mucositis due to previous cancer treatment } \\
\text { increases the risk }\end{array}$ \\
\hline
\end{tabular}

have summarized the patient-linked factors leading to increased risk for RIOM (Table 2) (15). They found that very young age, female gender, poor oral health and hygiene, decreased saliva secretion, low BMI, poor renal function with elevated serum creatinine level, smoking, and history of RIOM are risk factors predicting the development of RIOM in head and neck cancer patients (15).

\section{RIOM IMPACT AND SIDE EFFECTS}

Radiation-induced oral mucositis side effects and sequels include oral pain in $69 \%$ of patients, dysphagia in $56 \%$ of patients, opioid use in $53 \%$ of patients, weight loss of $3-7 \mathrm{~kg}$, feeding tube 
insertion and hospitalization (ICU admission) in 15\% of patients, and modification or interruption of treatment in $11-16 \%$ of patients $(1,12,16)$.

In the United States, RIOM may add up to $1,700.00-6,000.00$ USD per patient depending on the inflammatory grade of the injury (12). RIOM treatment adds an economic cost that was estimated to increase up to 17,000.00 USD per patient treated for head and neck cancers (16).

Radiation-induced oral mucositis injury challenges radiation oncologists from many aspects, such as radiation dose limitations, changes in dose fractionation protocol, and dramatic negative effects on patients' quality of life (1). The major clinical consequences of RIOM include hospital admission or extended hospitalization for total parenteral nutrition, intravenous (IV) analgesia, and IV antibiotics. Sixty-two percent of patients require hospitalization, and $70 \%$ of patients with grade $3-4$ oral mucositis $(\mathrm{OM})$ require feeding tube insertion. Reduction or cessation of cancer treatment occurs in $35 \%$ of patients due to the developed dose-limiting toxicity (17).

\section{PATHOGENESIS AND SUGGESTED MECHANISTIC PATHWAYS}

The pathophysiology of RIOM is not fully understood. Recent studies proposed that the pathogenesis of RIOM is composed of four phases: an initial inflammatory/vascular phase, an epithelial phase, a (pseudomembranous) ulcerative/bacteriological phase, and a healing phase $(2,5)$.

At the inflammatory phase, the RT-induced tissue injury results in the release of inflammatory cytokines; e.g., interleukin (IL)-1 $\beta$, prostaglandins (PGs), and tumor necrosis factor- $\alpha$ (TNF- $\alpha$ ) from the resident cells such as epithelial, endovascular, and connective tissue. These mediators might increase the damage by increasing the vascular permeability, leading to more infiltration and recruitment of inflammatory cells. Stem cells travel to the site of the tissue injury with other innate immunity components, e.g., MPO-positive leukocytes, macrophages, and neutrophils (18). On the other hand, there are some anti-inflammatory cytokines, such as IL-10 and IL-11, that work to minimize the injury (18).

The epithelial phase is initiated within a week by the apoptotic and cytotoxic effects of RT on the proliferating basal cells. This is why the recovery period is dependent on the rate of epithelial turnover, which could be enhanced by growth factors like epidermal growth factor and keratinocyte growth factor (KGF) (19).

After a week, the epithelial breakdown ends with the beginning of the ulceration. This occurs, when epithelial loss leads to disrupted basement membrane, formation of ulcer pseudomembrane, and inflammatory exudate. The ulceration stage is very painful, since the protective barrier that covers the nerve endings at the lamina propria is lost (19). The resulting microcoagulation and neutropenic state facilitate the Gram-negative bacteria and yeast colonization with the production of secondary infection. Bacterial exotoxins aggravate the inflammatory reaction by inducing mononuclear burst with the release of more IL-1 $\beta$, TNF- $\alpha$, and nitric oxide $(8,9,16,20)$.
TABLE 3 | Signaling pathways involved in the development of mucositis (10).

B-cells receptor signaling

Cell cycle: G2/M DNA damage checkpoint receptor

Death receptor signaling

Glutamate receptor signaling

Interleukin-6 signaling

Integrin signaling

Nuclear factor- $\mathrm{\kappa} B$ signaling

Nitrogen metabolism

PI3K/AKT signaling

P38 mitogen-activated protein kinase signaling

SAPK/JNK signaling

Toll-like receptor signaling

Vascular endothelial growth factor signaling

Wnt/B-catenin signaling

Signaling pathways suggested to be involved in RIOM pathobiology include nitrogen metabolism, Toll-like receptor signaling, nuclear factor- $\mathrm{B}$ (NF- $\kappa \mathrm{B})$ signaling, B-cell receptor signaling, P13K/AKT signaling, cell cycle: G2/M DNA damage checkpoint receptor, p38 mitogen-activated protein kinase (MAPK) signaling, Wnt/B-catenin signaling, glutamate receptor signaling, integrin signaling, vascular endothelial growth factor signaling, IL-6 signaling, death receptor signaling, and SAPK/ JNK signaling (Table 3) (10, 19).

In 2004, Sonis suggested five stages (phases) of OM injury induced by radiotherapy (RT) and/or CT: initiation, signaling, amplification, ulceration, and healing (Figure 1) (16).

In 2009, Redding summarized Sonis' RIOM pathobiology phases (Figure 2). The initiation phase with RT and/or CT injury results in direct and lethal DNA damage with the release of reactive oxygen species (ROS) from epithelial and vascular endothelial cells, fibroblasts, and tissue macrophages. This is followed by amplification of this signal (11). During the primary damage response, the DNA damage and ROS act through three major pathways: (1) fibronectin breakdown, which stimulates the macrophages leading to activation of the matrix metalloproteinases (MMPs), (2) nuclear factor $-\kappa \mathrm{B}(\mathrm{NF}-\kappa \mathrm{B})$ activation, which stimulates the gene expression and the release of proinflammatory cytokines, e.g., TNF- $\alpha$, IL- $1 \beta$, and IL-6, and (3) ceramide pathway through sphingomyelinase and ceramide synthase. The end result will be more tissue injury and stimulated apoptosis (11). During the signal amplification phase, there is restimulation of tissue damage and apoptosis by the major pro-inflammatory cytokines (TNF- $\alpha$, IL- $1 \beta$, and IL-6), NF- $\kappa \mathrm{B}$ mediated gene expression, and ceramide and caspase pathways. The basement membrane protective barrier is lost during the ulceration phase. This leads to Gram-negative and yeast secondary infection potential, which adds more pro-inflammatory reactions and complicates the already existing inflammation. The healing phase starts by matrix signaling to basal epithelial cells to migrate, proliferate, and differentiate (11). Signal amplification during RIOM or CT-induced OM is a main step in this treatment-induced injury, according to Sonis et al. (17). RT and/or CT activate the transcription factor NF- $\kappa \mathrm{B}$ in epithelial, endothelial, and mesenchymal cells and macrophages, resulting in upregulation of genes and production of pro-inflammatory 

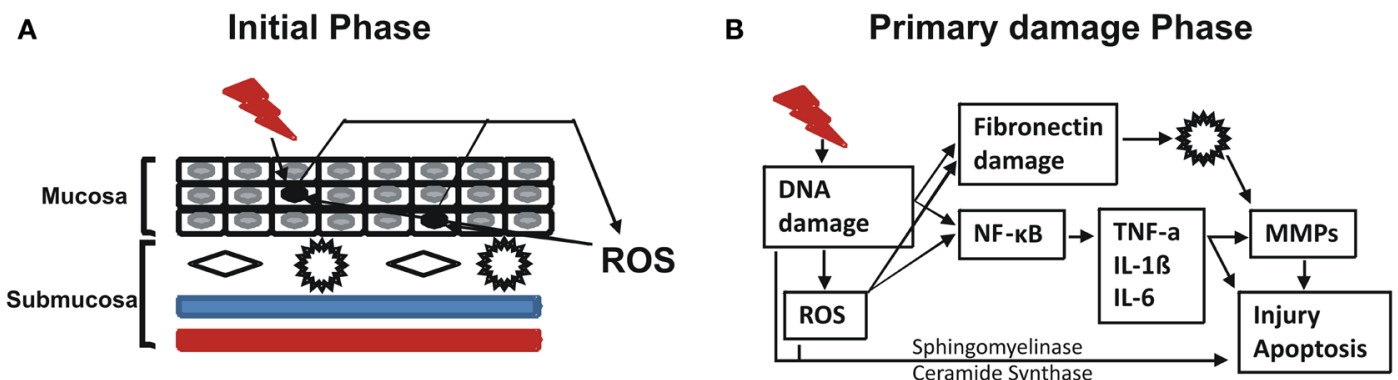

C Signal Amplification Phase
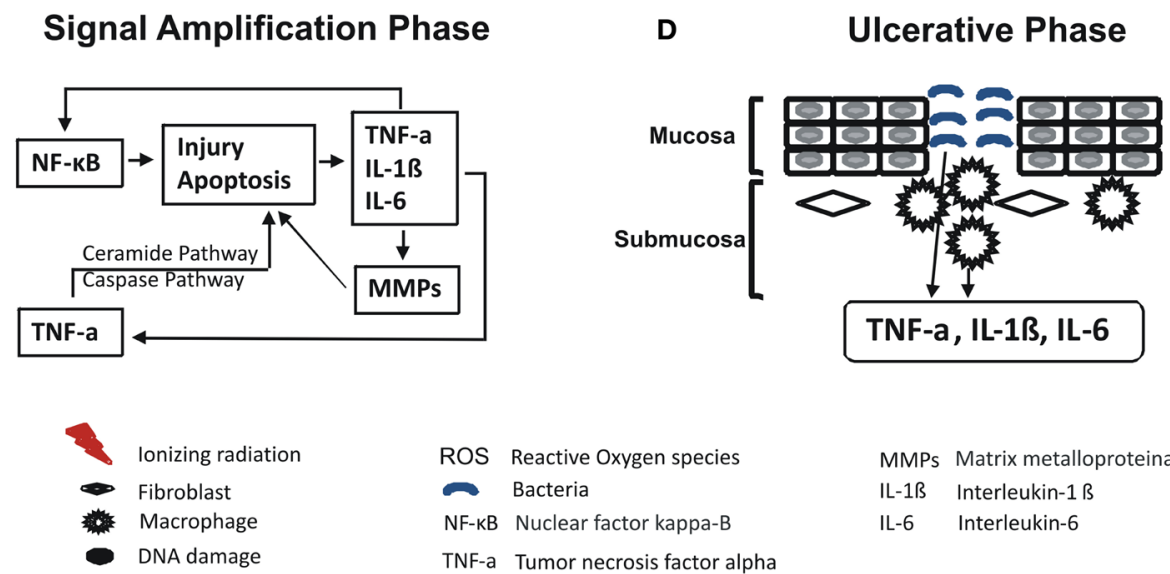

$$
\begin{aligned}
& \text { ROS Reactive Oxygen species } \\
& \text { NF-KB Nuclear factor kappa-B } \\
& \text { TNF-a Tumor necrosis factor al pha }
\end{aligned}
$$

FIGURE 2 | Redding's summary of RT and/or chemotherapy (CT)-induced oral mucositis pathobiology (11). Redding has summarized the pathobiology phases of radiation-induced oral mucositis induced by RT and/or CT. In brief, initiation phase with RT and/or CT results in direct and lethal DNA damage, which leads to release of reactive oxygen species (ROS) from epithelial, vascular endothelial, fibroblasts, and tissue macrophages with cycles of amplifications. Within such primary damage response, the DNA damage and ROS lead to three major steps: (1) fibronectin breakdown that activates macrophages ending with stimulation of matrix metalloproteinase; (2) nuclear factor-kB (NF-kB) activation that stimulates the gene expression and release of pro-inflammatory cytokines, e.g., TNF- $\alpha$, interleukin (IL)-1 $\beta$, and IL-6; and (3) ceramide pathway through sphingomyelinase and ceramide synthase. The result will be more tissue injury and stimulated apoptosis. During the signal amplification phase, there is restimulation of tissue damage and apoptosis by the major pro-inflammatory cytokines (TNF- $\alpha$, IL- $1 \beta$, and (L-6), NF-kB-mediated gene expression, and ceramide and caspase pathways. During the ulceration and loss of the protective barrier, secondary infection adds more pro-inflammatory reactions and complicates the already existing inflammation before the healing phase starts by matrix signaling to basal epithelial cells to migrate, proliferate, and differentiate. Republished with the permission of Dr. Redding. (A) Initial phase, (B) primary damage phase, (C) signal amplification phase, and (D) ulcerative phase.

cytokines: tumor necrosis factor- $\alpha(\mathrm{TNF}-\alpha)$ and IL- $1 \beta$, which amplify the primary signal and activate NF- $\mathrm{B}$. This leads to transcription of genes responsible for MAPK, cyclooxegenase- 2 (COX-2), and tyrosine kinase signaling molecules. These signaling pathways activate MMPs 1 and 3 in the epithelial and lamina propria cells, which collectively cause tissue injury (10) (Figure 3).

\section{RIOM GRADING AND SCORING SCALES}

There has been more than one grading scale for RIOM. Table 4 shows the comparison of different RIOM scoring scales (14,21-23).

World Health Organization Oral Toxicity Scale measures the anatomical, symptomatic, and functional elements of $\mathrm{OM}$ (Figure 4). The Radiation Therapy Oncology Group (RTOG) determined the acute radiation morbidity scoring criteria for mucous membranes. Finally, the Western Consortium for Cancer Nursing Research describes only the anatomical changes associated with OM (24).
Radiation Therapy Oncology Group developed the Acute Radiation Morbidity Scoring Criteria for the evaluation of RT effects (another criterion was generated for late effects of RT) (25). The National Cancer Institute (NCI) Common Toxicity Criteria (NCI-CTC) scores CT-related side effects. The RTOG was gathered with the NCI-CTC to produce version 2.0, which has been used in all NCI clinical trials since March 1998 (Table 5) $(2,25,26)$.

The OM Index (OMI) scores the severity of OM by the erythema, ulceration, atrophy, and edema (a scale of $0-3$ was designated for each element: $0=$ none and 3 = severe). The OMI is considered internally consistent with high test-retest and interscorer reliability, and it shows solid validity (27).

The OM Assessment Scale (OMAS) is a highly reproducible scoring scale for RIOM, responsive over time, and accurate in detecting OM-associated elements (25). OMAS records the objective assessment of OM depending on the scoring of the presence and size of the ulceration or pseudomembrane (score 0-3: $0=$ no lesion; $1=$ lesion of $<1 \mathrm{~cm}^{2} ; 2=$ lesion of $1-3 \mathrm{~cm}^{2}$; 
$3=$ lesion $>3 \mathrm{~cm}^{2}$ ) and erythema (score 0-2: $0=$ none; $1=$ not severe; 2 = severe) on the upper and lower lips, right and left cheeks, right and left ventral and lateral tongue, floor of the mouth, soft palate, and hard palate $(23,28)$.

All these scoring scales are validated and are required in assessing RIOM and the therapeutic benefits of any new treatment of RIOM.

\section{DIAGNOSIS OF RIOM}

Radiation-induced oral mucositis can develop within or after 2 weeks from the beginning of RT. Oral assessment guide could be a useful tool for detection of early OM (Table 6) (20). Apart from the early clinical signs and symptoms, CBC with differential is considered the baseline to help radiation oncologists to determine the most susceptible time for developing OM or oral infection. Radiation oncologists can start the RT provided that there is no evidence of any periodontal disease. If at any point

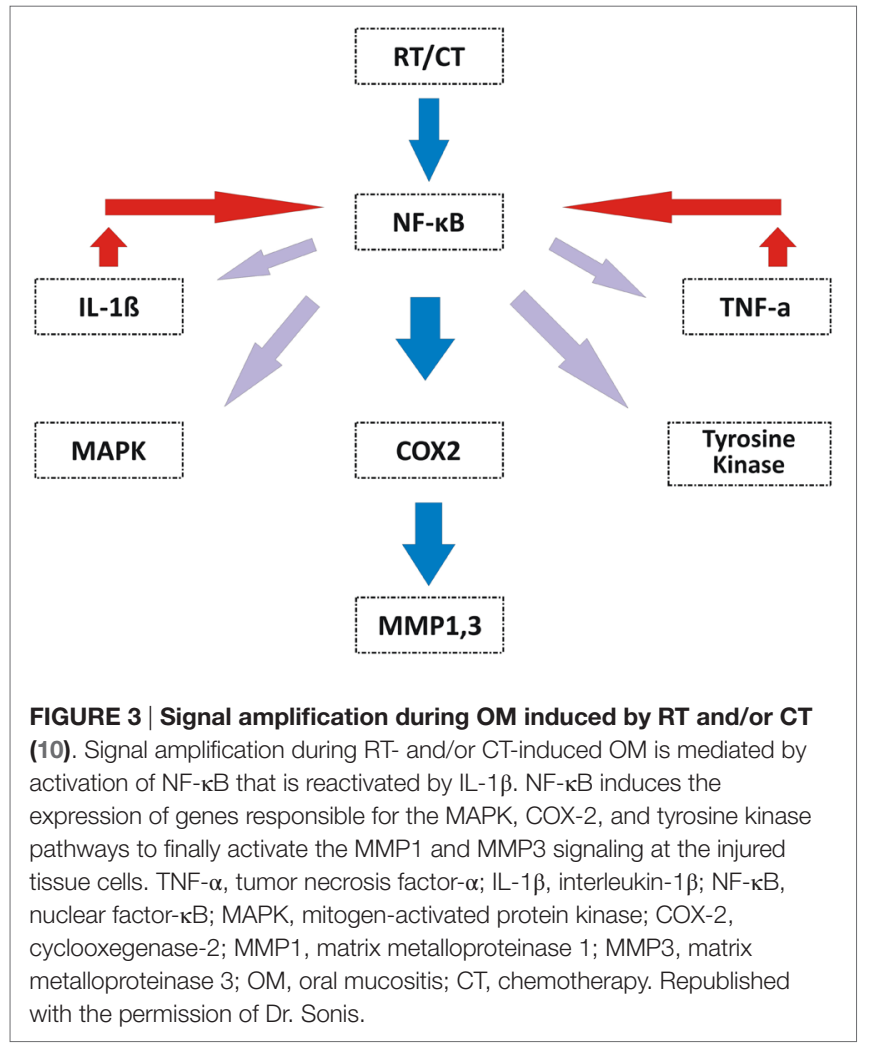

RIOM develops, oral lesion culture and antimicrobial therapy are recommended as soon as possible (29). Since renal diseases are considered contributing factors for OM (15), chemistry levels should be regularly monitored by the treating physician (29).

\section{DIFFERENTIAL DIAGNOSIS OF RIOM}

Because similar conditions can coexist in immunocompromised patients including cancer patients receiving RT and/or CT, differential diagnosis for RIOM is critical. Table 7 shows possible similar conditions (Figure 5) (20, 31).

\section{PROGNOSIS OF RIOM}

The general long-term prognosis is reasonably good since most lesions resolve within 2-4 weeks after stopping the RT or CT. Although RIOM is considered a self-limited injury in some patients, it could be a lethal injury in moderately to severely ill patients, which could lead to ICU admission with obligatory cessation of RT. Patient losses are a common event under these circumstances (32).

\section{PREVENTION OF RIOM}

Maintaining good oral care is the main preventive measure for RIOM to minimize the risk for candidiasis or secondary bacterial infection, especially in hyperfractionated radiotherapy, combined CCRT regimens, or RT combined with a targeted agent due to increased mucositis severity (3). We will summarize the most recent agents and measures to prevent RIOM.

1. Good oral hygiene

Good oral hygiene has been found to be one of the most effective ways to lower the risk of RIOM and minimize its progression. Pre-existing oral pathology, e.g., dental caries, periodontal lesions, pulpal disease, and oral xerostomia, has been linked with increased bacteria colonization and severe RIOM. It is recommended to do early oral examination before starting any mucosal toxic therapy for cancer patients. To help minimize the oral side effects of antineoplastic therapy, it is recommended to eliminate any oral pathology before the beginning of RT. This may be accomplished by performing early histological, cytological, microbiologic, and serologic examinations (2). The Multinational Association of Supportive Care in Cancer (MASCC) and the International Society of Oral Oncology (ISOO) guidelines

TABLE 4 | Comparison of OM scoring scales (14, 21-23).

\begin{tabular}{|c|c|c|c|c|c|}
\hline Grade & 0 & 1 & 2 & 3 & 4 \\
\hline $\mathrm{WHO}$ & None & Soreness \pm erythema & $\begin{array}{l}\text { Erythema, ulcers, and patient can } \\
\text { swallow solid food }\end{array}$ & $\begin{array}{l}\text { Ulcers with extensive erythema and patient } \\
\text { cannot swallow solid food }\end{array}$ & $\begin{array}{l}\text { mucositis to the extent that } \\
\text { alimentation is not possible }\end{array}$ \\
\hline RTOG & None & Erythema of the mucosa & $\begin{array}{l}\text { Patchy reaction }<1.5 \mathrm{~cm} \text {, } \\
\text { non-contiguous }\end{array}$ & Confluent reaction $>1.5 \mathrm{~cm}$, contiguous & $\begin{array}{l}\text { Necrosis or deep } \\
\text { ulceration, } \pm \text { bleeding }\end{array}$ \\
\hline \multirow[t]{3}{*}{ WCCNR } & Lesions: none & Lesions: 1-4 & Lesions: >4 & Lesions: coalescing & $\mathrm{N} / \mathrm{A}$ \\
\hline & Color: pink & Color: slight red & Color: moderate red & Color: very red & \\
\hline & Bleeding: none & Bleeding: N/A & Bleeding: spontaneous & Bleeding: spontaneous & \\
\hline
\end{tabular}

WHO, World Health Organization; RTOG, Radiation Therapy Oncology Group; WCCNR, Western Consortium for Cancer Nursing Research; OM, oral mucositis. 


\section{World Health Organization's Oral Toxicity Scale}
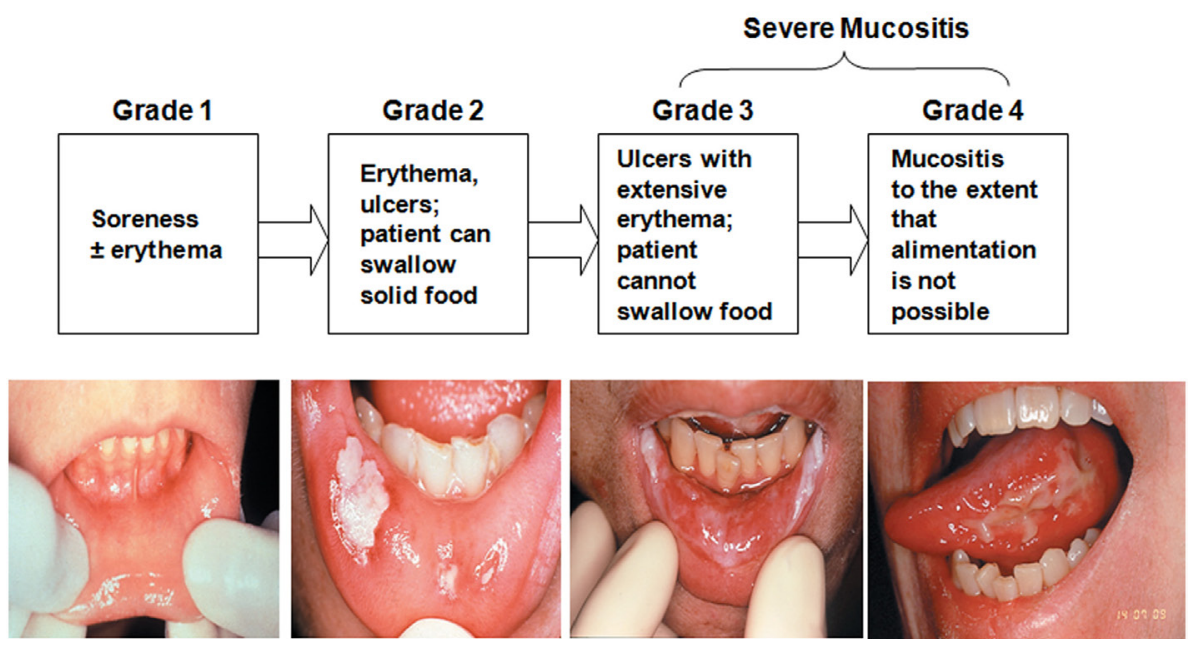

FIGURE 4 | World Health Organization's Oral Toxicity Scale. Republished with the permission of Dr. Patrick Stiff, Loyola University Medical Center, Maywood, IL, USA.

TABLE 5 | Toxicity grading of oral mucositis (OM) according to World Health Organization (WHO) and National Cancer Institute Common Toxicity Criteria (NCI-CTC) criteria (2)a.

\begin{tabular}{|c|c|c|c|c|c|}
\hline Side effect & $\begin{array}{l}\text { Grade } 0 \\
\text { (none) }\end{array}$ & $\begin{array}{l}\text { Grade } 1 \\
\text { (mild) }\end{array}$ & $\begin{array}{l}\text { Grade } 2 \\
\text { (moderate) }\end{array}$ & $\begin{array}{l}\text { Grade } 3 \\
\text { (severe) }\end{array}$ & $\begin{array}{l}\text { Grade } 4 \\
\text { (life threatening) }\end{array}$ \\
\hline $\begin{array}{l}\text { WHO oral mucositis } \\
\text { (stomatitis) }\end{array}$ & None & $\begin{array}{l}\text { Oral soreness, } \\
\text { erythema }\end{array}$ & $\begin{array}{l}\text { Oral erythema, ulcers, can } \\
\text { eat solids }\end{array}$ & Oral ulcers, requires liquid diet only & Oral alimentation not possible \\
\hline $\begin{array}{l}\mathrm{NCl}-\mathrm{CTC} \text { chemotherapy- } \\
\text { induced stomatitis/ } \\
\text { pharyngitis (oral/ } \\
\text { pharyngeal mucositis) }\end{array}$ & None & $\begin{array}{l}\text { Painless ulcers, } \\
\text { erythema, or mild } \\
\text { soreness in the } \\
\text { absence of lesions }\end{array}$ & $\begin{array}{l}\text { Painful erythema, edema, } \\
\text { or ulcers, but can eat or } \\
\text { swallow }\end{array}$ & $\begin{array}{l}\text { Painful erythema, edema, or ulcers } \\
\text { requiring intravenous hydration }\end{array}$ & $\begin{array}{l}\text { Severe ulceration or requires } \\
\text { parenteral or enteral nutritional support } \\
\text { or prophylactic intubation }\end{array}$ \\
\hline $\begin{array}{l}\mathrm{NCl}-\mathrm{CTC} \text { mucositis due to } \\
\text { radiation }\end{array}$ & None & $\begin{array}{l}\text { Erythema of the } \\
\text { mucosa }\end{array}$ & $\begin{array}{l}\text { Patchy } \\
\text { pseudomembranous } \\
\text { reaction (patches generally } \\
\leq 1.5 \mathrm{~cm} \text { in diameter and } \\
\text { non-contiguous) }\end{array}$ & $\begin{array}{l}\text { Confluent pseudomembranous } \\
\text { reaction (contiguous patches } \\
\text { generally }>1.5 \mathrm{~cm} \text { in diameter) }\end{array}$ & $\begin{array}{l}\text { Necrosis or deep ulceration; may } \\
\text { include bleeding not induced by minor } \\
\text { trauma or abrasion }\end{array}$ \\
\hline $\begin{array}{l}\text { NCI-CTC stomatitis/ } \\
\text { pharyngitis (oral/ } \\
\text { pharyngeal mucositis) } \\
\text { for bone marrow }\end{array}$ & None & $\begin{array}{l}\text { Painless ulcers, } \\
\text { erythema, or mild } \\
\text { soreness in the } \\
\text { absence of lesions }\end{array}$ & $\begin{array}{l}\text { Painful erythema, edema, } \\
\text { or ulcers, but can swallow }\end{array}$ & $\begin{array}{l}\text { Painful erythema, edema, or ulcers } \\
\text { preventing swallowing or requiring } \\
\text { hydration or parenteral (or enteral) } \\
\text { nutritional support }\end{array}$ & $\begin{array}{l}\text { Severe ulceration requiring } \\
\text { prophylactic intubation or resulting in } \\
\text { documented aspiration pneumonia }\end{array}$ \\
\hline
\end{tabular}

transplantation studies

${ }^{a}$ Republished with the permission of Dr. Christoph C. Zielinski.

recommend the use of a standardized oral care protocol, e.g. brushing with a soft toothbrush, flossing and the use of non-medicated rinses (saline or sodium bicarbonate rinses)

(Table 9) (33-36). The good oral care can be summarized as follows:

- Rinsing with a non-irritating solution, e.g., saline to increase the quality of saliva.

- Daily ultrasoft tooth brushing with fluoride toothpaste.

- Scaling and cleaning.

- Very soft diet with low sugar and non-acidic food and drinks (Table 8).

- Flossing is not recommended due to low platelet count.

- Minimize denture use.
- No smoking or alcohol.

- Other preventive procedures include minimizing the microbial load (will be discussed more in the treatment section) and educating the patient on good oral hygiene, which is mandatory.

2. Cryotherapy has been recommended for CT-induced OM, but no proven role in RIOM due to insufficient evidence (33).

3. Keratinocyte growth factor is an epithelial mitogen that reduces the levels of ROS by activating nuclear factor (erythroidderived 2)-like 2 and had been used in RIOM with promising results (37-53). It appears to be one of the promising treatment and prevention options for RIOM that has been investigated in clinical trials $(39,43)$. Palifermin (IV recombinant human 
KGF-1) had been approved by the US-FDA for minimizing $\mathrm{OM}$ in hematologic malignancies' patients who receive myelotoxic therapies and require hematopoietic cell support after its reliable results in alleviating $\mathrm{WHO}$ grade 3 and $4 \mathrm{OM}$ in these patients. Palifermin is delivered IV 3 days before of CT/RT and for 3 days after CT. Palifermin should be avoided on the same day of CT/RT (33).

4. Amifostine is a free-radical scavenger, antioxidant, and cytoprotective agent that was administered subcutaneously (SC) and IV in many clinical trials for RIOM. Amifostine is conventionally given IV before RT or CT. It is approved by the US-FDA to reduce the cumulative renal toxicity associated with repeated administration of cisplatin in patients with advanced ovarian cancer. In addition, it was approved for by the US-FDA to reduce the incidence of moderate to severe xerostomia in patients undergoing postoperative RT for head and neck cancer, where the radiation port includes a substantial portion of the parotid glands $(33,54-62)$. Although there was a reduction in the pro-inflammatory cytokine production, its side effects, e.g., hypotension and nausea, were recorded, particularly with IV route. Nevertheless, SC injection 60 min before RT in head and neck cancer patients

\begin{tabular}{|c|c|c|c|}
\hline $\begin{array}{l}\text { Item/ } \\
\text { grade }\end{array}$ & 1 & 2 & 3 \\
\hline Voice & Normal & Deeper or raspy & Difficulty talking \\
\hline Swallow & Normal & Some pain & Unable to swallow \\
\hline Lips & $\begin{array}{l}\text { Smooth pink } \\
\text { and moist }\end{array}$ & Dry or cracked & Ulcerated or bleeding \\
\hline Tongue & $\begin{array}{l}\text { Pink and } \\
\text { moist }\end{array}$ & $\begin{array}{l}\text { Coated and } \\
\text { shiny } \pm \text { red }\end{array}$ & Blistered or cracked \\
\hline Saliva & Watery & Thick & Absent \\
\hline $\begin{array}{l}\text { Mucus } \\
\text { membrane }\end{array}$ & $\begin{array}{l}\text { Pink and } \\
\text { moist }\end{array}$ & $\begin{array}{l}\text { Red and coated } \\
\text { without ulcers }\end{array}$ & Ulcers \\
\hline Gingiva & Pink and firm & Edematous \pm redness & $\begin{array}{l}\text { Spontaneous or pressure- } \\
\text { induced bleeding }\end{array}$ \\
\hline $\begin{array}{l}\text { Teeth/ } \\
\text { denture } \\
\text { areas }\end{array}$ & $\begin{array}{l}\text { Clean, no } \\
\text { debris }\end{array}$ & $\begin{array}{l}\text { Plaque and localized } \\
\text { debris }\end{array}$ & $\begin{array}{l}\text { Generalized plaque or } \\
\text { debris }\end{array}$ \\
\hline
\end{tabular}

showed marked reduction of these side effects, unfortunately, with reduced efficacy and patient compliance. Only cutaneous toxicity was noted in SC route of amifostine delivery $(54,55)$. For moderate to severe RT-induced xerostomia in head and neck cancer patients, the recommended dose of amifostine is $200 \mathrm{mg} / \mathrm{m}^{2}$ once daily over $3 \mathrm{~min} \mathrm{IV,} \mathrm{starting}$ 15-30 min before standard fraction RT (1.8-2.0 Gy). Blood pressure should be monitored before, during, and after the IV infusion. Oral 5-HT3 receptor antagonists with/without other antiemetics are recommended before amifostine therapy (63-65).

5. Radiation shields (intraoral devices), midline mucosa-sparing blocks, 3-D and RT field design, intensity-modulated radiation therapy (IMRT), and removal of separable prosthetics are shown in preclinical studies to reduce the radiation scatter and the RIOM injury (66-69).

6. Low-energy helium-neon laser applied before RT showed significant reduction in the duration and the severity of RIOM in head and neck cancer patients (70). MASCC/ISOO guidelines suggest the use of low-level laser therapy in CT-induced $\mathrm{OM}$ at centers that can provide the necessary technology and training (33).

\section{SYMPTOMATIC TREATMENT OF RIOM}

No single agent has been approved by the US-FDA for the treatment of RIOM. Symptoms reduction and complications prevention of RIOM, including nutritional support, pain control, prophylaxis, and/or treatment of secondary infections, are considered the main cornerstone in the management of RIOM (34-36). Agents that were investigated and/or applied in RIOM treatment are discussed in the context of recently updated evidence-based preclinical and clinical studies.

\section{Locally applied agents}

1. Glycyrrhetinic acid/povidone/sodium hyaluronate gel has mechanical action implemented in the relief of pain in RIOM. It adheres to the mucosal surface of the mouth, soothing oral lesions. Nevertheless, the preclinical studies

TABLE 7 | Differential diagnosis of RIOM $(20,31)$.

\begin{tabular}{|c|c|c|c|c|}
\hline Disease/injury & Cause & Clinical presentation/lab findings & Severity & Treatment options \\
\hline Oral mucositis & $\begin{array}{l}\text { Chemotherapy and } \\
\text { radiation therapy }\end{array}$ & $\begin{array}{l}\text { Diffuse redness, ulcerations, and pain, particularly } \\
\text { in areas where teeth abut tissue }\end{array}$ & $\begin{array}{l}\text { Varies; in BMT setting up } \\
\text { to } 98 \% \text { have grade } 3 / 4\end{array}$ & $\begin{array}{l}\text { Palliative rinses, narcotics, palifermin in } \\
\text { the BMT setting }\end{array}$ \\
\hline Aphthous stomatitis & Etiology not identified & Single painful ulcer & $\begin{array}{l}\text { Localized, but painful; } \\
\text { maximum grade } 2\end{array}$ & Topical \\
\hline Herpetic mucositis & HSV1 & Usually several spots; ulcerative & Usually grade $1-2$ & Acyclovir, valacyclovir, foscarnet \\
\hline Oral thrush & Candida & $\begin{array}{l}\text { Varies from painless to mild soreness; whitish } \\
\text { plaques }\end{array}$ & Usually grade $0-1$ & $\begin{array}{l}\text { Nystatin rinses; fluconazole and other } \\
\text { azoles }\end{array}$ \\
\hline Denture/oral trauma & Dentures & $\begin{array}{l}\text { Common in elderly patients with loose-fitting } \\
\text { dentures }\end{array}$ & Can limit calories & Repair, removal of dentures \\
\hline $\begin{array}{l}\text { Gangrenous } \\
\text { stomatitis }\end{array}$ & Bacterial infections & Necrotic pseudomembranes & Rare, can be severe & $\begin{array}{l}\text { Antibacterials that treat oral aerobes and } \\
\text { anaerobes }\end{array}$ \\
\hline $\begin{array}{l}\text { Acute necrotizing } \\
\text { stomatitis }\end{array}$ & $\begin{array}{l}\text { Bacterial infections } \\
\text { in immune-deficient } \\
\text { patients }\end{array}$ & Pain, fever, necrotic, bloody ulcers & Grade 3/4 & Control of infection \\
\hline
\end{tabular}

BMT, bone marrow transplantation; RIOM, radiation-induced oral mucositis; HSV1, herpes simplex virus type 1. 

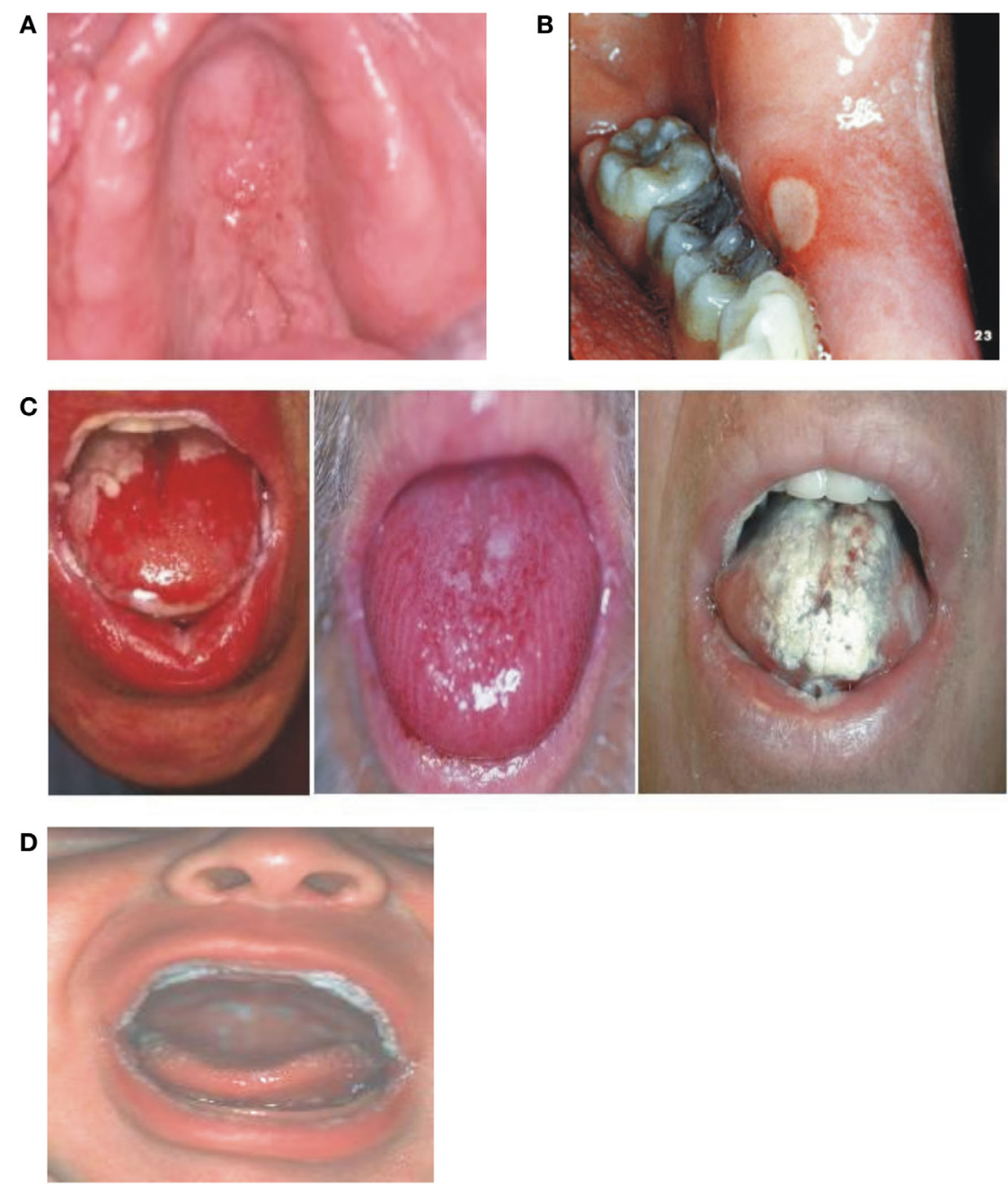

FIGURE 5 | Differential diagnosis of radiation-induced oral mucositis. Republished with the permission of Dr. Patrick Stiff, Loyola University Medical Center, Maywood, IL, USA. (A) Local, denture-related lesion, (B) aphthous ulcer, (C) oral mucositis, and (D) oral thrush.

TABLE 8 | Diet recommended for RIOM patients (20).

\begin{tabular}{lll}
\hline Typically accessed diet & Things to avoid & $\begin{array}{l}\text { Habits to } \\
\text { avoid }\end{array}$ \\
\hline Liquids & $\begin{array}{l}\text { Rough food (potato chips, crisps, toast) } \\
\text { Spices }\end{array}$ & $\begin{array}{l}\text { Smoking } \\
\text { Alcohol }\end{array}$ \\
lce & Salt & \\
Custards & Acidic fruit (grapefruit, lemon, orange) & \\
Non-acidic fruits (banana, & & \\
mango, melon, peach) & & \\
Soft cheeses & & \\
Eggs & &
\end{tabular}

are controversial, and only one clinical trial on unknown results was conducted to date (71).

2. L-Glutamine is a non-essential amino acid that counteracts RT-induced metabolic deficiencies (72). Locally applied L-glutamine reduced the RIOM in a randomized clinical trial (73). Glutamine powder for oral suspension was approved by the US-FDA for topical application in management of CT-induced OM, mainly IOMyet (74).

3. Manganese superoxide dismutase is a detoxifying agent that removes ROS. It was shown to have radioprotective effects against RT-induced colitis, esophagitis, hepatic cells apoptosis, and intestinal and eye injury (75-98). Phase I dose escalation study of GC4419 (manganese-containing macrocyclic ligand complex similar to naturally occurring superoxide dismutase enzymes) in combination with CT/RT for squamous cell cancer of the head and neck has just been completed waiting for results release (NCT01921426).

4. Local anesthetics, e.g., diphenhydramine, viscous xylocaine, lidocaine, and dyclonine hydrochloride, are used for short-term relief of pain associated with RIOM, despite the fact that they can interfere with the taste sensation leading to hypoalimentation $(99,100)$. Swishing and gargling the anesthetic viscous gel containing $2 \%$ 
TABLE 9 | Multinational Association for Supportive Care in Cancer/International Society for Oral Oncology (MASCC/ISOO) Clinical Practice Guidelines for oral mucositis (3).

Recommendations in favor of an intervention (strong evidence supports effectiveness in the treatment setting listed)

Oral cryotherapy for $30 \mathrm{~min}$

Recombinant human keratinocyte growth factor-1 (palifermin) at a dose of $60 \mu \mathrm{g} / \mathrm{kg}$ per day for 3 days prior to conditioning treatment and for 3 days after transplant

Low-level laser therapy (wavelength at $650 \mathrm{~nm}$, power of $40 \mathrm{~mW}$, and each square centimeter treated with the required time to a tissue energy dose of $2 \mathrm{~J} / \mathrm{cm}^{2}$ )

Patient-controlled analgesia with morphine

Benzydamine mouthwash
Prevention of OM Patients receiving bolus 5-fluorouracil chemotherapy

Prevention of OM Patients receiving high-dose chemotherapy and TBI, followed by autologous stem cell transplantation, for a hematological malignancy

Prevention of OM Patients receiving HSCT conditioned with high-dose chemotherapy, with or without TBI

Pain reduction Patients undergoing HSCT

Level II

Prevention of OM Patients with HNC receiving moderate dose radiation therapy (up to 50 Gy), Level II without concomitant chemotherapy

Level II

Level II

Level II
Suggestions in favor of an intervention (weaker evidence supports effectiveness in the treatment setting listed)
Oral care protocols

Oral cryotherapy

Low-level laser therapy (wavelength around $632.8 \mathrm{~nm}$ )
Prevention of $\mathrm{OM}$

Prevention of $\mathrm{OM}$ evel III

Level III
conditioning for HSCT

Prevention of OM Patients undergoing radiotherapy, without concomitant chemotherapy, Level III for $\mathrm{HNC}$

\begin{tabular}{|c|c|c|c|}
\hline Transdermal fentanyl & Pain reduction & $\begin{array}{l}\text { Patients receiving conventional or high-dose chemotherapy, with or } \\
\text { without TBI }\end{array}$ & Level III \\
\hline $2 \%$ morphine mouthwash & Pain reduction & Patients receiving chemoradiation for $\mathrm{HNC}$ & Level III \\
\hline 0.5\% doxepin mouthwash & Pain reduction & All patients with OM-induced pain & Level IV \\
\hline Systemic zinc supplements administered orally & Prevention of $\mathrm{OM}$ & HNC patients receiving radiation therapy or chemoradiation & Level III \\
\hline
\end{tabular}

\section{Recommendations against interventions (strong evidence indicates lack of effectiveness in the treatment setting listed)}

PTA (polymyxin, tobramycin, amphotericin B) and BCoG Prevention of OM Patients receiving radiation therapy for HNC

(bacitracin, clotrimazole, gentamicin)

Iseganan antimicrobial mouthwash

Prevention of OM Patients receiving high-dose chemotherapy, with or without TBI, for

Level II

HSCT or in patients receiving radiation therapy or concomitant

chemoradiation for HNC

Iseganan antimicrobial mouthwash

Prevention of $\mathrm{OM}$ Patients receiving high-dose chemotherapy, with or without $\mathrm{TBI}$, for Level II HSCT or in patients receiving radiation therapy or concomitant chemoradiation for HNC

Sucralfate mouthwash

Prevention of OM Patients receiving chemotherapy for cancer (I), or inpatients receiving radiation therapy (I) or concomitant chemoradiation (II) for HNC

Sucralfate mouthwash

Treatment of $\mathrm{OM}$ Patients receiving chemotherapy for cancer (I), or in patients receiving radiation therapy (II) for HNC

Intravenous glutamine

Prevention of OM Patients receiving high-dose chemotherapy, with or without TBI, for HSCT

Level I, II

Suggestions against interventions (weaker evidence indicates lack of effectiveness in the treatment setting listed)

Chlorhexidine mouthwash

Prevention of $\mathrm{OM}$ Patients receiving radiation therapy for $\mathrm{HNC}$

Level III

Granulocyte-macrophage colony-stimulating factor mouthwash

Prevention of $\mathrm{OM}$ Patients receiving high-dose chemotherapy, for autologous or

Level II allogeneic HSCT

Misoprostol mouthwash

Systemic pentoxifylline, administered orally

Prevention of $\mathrm{OM}$ Patients receiving radiation therapy for $\mathrm{HNC}$

Level III

Prevention of OM Patients undergoing HSCT

Level III

Systemic pilocarpine, administered orally

Prevention of OM Patients receiving radiation therapy for head and neck cancer (III), or patients receiving high-dose chemotherapy, with or without TBI, for HSCT (II)

OM, oral mucositis; HSCT, hematopoietic stem cell transplantation; TBI, total body irradiation; HNC, head and neck cancer.

Criteria for each level of evidence (34).

Level l: evidence obtained from meta-analysis of multiple, well-designed, controlled studies; randomized trials with low false-positive and false-negative errors (high power).

Level Il: evidence obtained from at least one well-designed experimental study; randomized trials with high false-positive and/or false-negative errors (low power).

Level III: evidence obtained from well-designed, quasi-experimental studies such as non-randomized, controlled single-group, pretest-posttest comparison, cohort, time, or matched case-control series.

Level IV: evidence obtained from well-designed, non-experimental studies, such as comparative and correlational descriptive and case studies.

Level V: evidence obtained from case reports and clinical examples. 
TABLE 10 | Radiation-induced oral mucositis (RIOM) the clinical trials that have been done until 2001 (2) .

\begin{tabular}{|c|c|c|c|c|c|}
\hline Injury & Reference & $\begin{array}{l}\text { Randomized/ } \\
\text { controlled/ } \\
\text { double blind }\end{array}$ & $\mathbf{P} / \mathbf{T}$ & Application/doses & Results \\
\hline $\mathrm{RT}$ & Shieh et al. (241) & Yes/yes/no & $\mathrm{P}$ & Instructions on oral care & Significant reduction \\
\hline RT & Perch et al. (68) & $\mathrm{No} / \mathrm{no} / \mathrm{no}$ & $P$ & Midline mucosa-sparing blocks & $\begin{array}{l}\text { Decreased mucositis without affecting } \\
\text { tumor control }\end{array}$ \\
\hline $\mathrm{RT}$ & Rugg et al. (242) & No/no/no & $P$ & Smoking during RT & Higher mucositis incidence in smokers \\
\hline $\mathrm{RT}$ & Scherlacher et al. (243) & Yes/yes/no & $P$ & Sucralfate vs. standard oral hygiene & $\begin{array}{l}\text { Significant reduction of incidence and } \\
\text { severity of mucositis }\end{array}$ \\
\hline RT & Allison et al. (146) & Yes/yes/no & $\mathrm{P}+\mathrm{T}$ & Sucralfate + fluconazole vs. standard oral care & $\begin{array}{l}\text { Significant reduced severity and } \\
\text { symptomatic relief }\end{array}$ \\
\hline $\mathrm{RT}$ & Franzen et al. (145) & Yes/yes/yes & $P$ & Sucralfate vs. placebo & $\begin{array}{l}\text { Significant lower incidence of severe } \\
\text { mucositis }\end{array}$ \\
\hline $\mathrm{RT}$ & Makkonen et al. (147) & Yes/yes/yes & $\mathrm{P}$ & Sucralfate vs. placebo & Only slight protective effect of sucralfate \\
\hline $\mathrm{RT}$ & Epstein et al. (148) & Yes/yes/yes & $P+T$ & Sucralfate vs. placebo & $\begin{array}{l}\text { Non-significant reduction of oral } \\
\text { discomfort }\end{array}$ \\
\hline $\mathrm{RT}$ & Meredith et al. (144) & Yes/yes/yes & $\mathrm{T}$ & Antacid, diphenhydramine, lidocaine \pm sucralfate & Non-significant reduction of severity \\
\hline $\mathrm{RT}$ & Cengiz et al. (142) & Yes/yes/yes & $\mathrm{P}+\mathrm{T}$ & Sucralfate vs. placebo & Decreased severity \\
\hline $\mathrm{RT}$ & Carter et al. (244) & Yes/yes/yes & $P$ & Sucralfate vs. placebo & No difference \\
\hline $\mathrm{RT}$ & Barker et al. (100) & Yes/yes/yes & $\mathrm{P}+\mathrm{T}$ & $\begin{array}{l}\text { Oral hygiene + sucralfate vs. } \\
\text { diphenhydramine + kaolin-pectin }\end{array}$ & No difference \\
\hline $\mathrm{RT}$ & Feber et al. (245) & Yes/yes/no & $\mathrm{P}$ & Hydrogen peroxide vs. saline & Significantly more oral discomfort \\
\hline $\mathrm{RT}$ & Spijkervet et al. (246) & Yes/yes/yes & $\mathrm{P}+\mathrm{T}$ & Chlorhexidine vs. placebo & No difference \\
\hline $\mathrm{RT}$ & Foote et al. (117) & Yes/yes/yes & $\mathrm{P}+\mathrm{T}$ & Chlorhexidine vs. placebo & Slight aggravation \\
\hline $\mathrm{HD}-\mathrm{CT}+\mathrm{RT}$ & Ferretti et al. (247) & Yes/yes/yes & $P+T$ & Chlorhexidine vs. placebo & $\begin{array}{l}\text { Significant reduction of incidence and } \\
\text { severity in the CT group only }\end{array}$ \\
\hline $\mathrm{CT}+\mathrm{RT}$ & Rahn et al. (157) & Yes/yes/no & $P$ & $\begin{array}{l}\text { Nystatin, rutosides, immuno-globuines, } \\
\text { panthenol } \pm \text { PVP-iodine }\end{array}$ & Significant reduction \\
\hline $\mathrm{CT}+\mathrm{RT}$ & Adamietz et al. (159) & Yes/yes/no & $P$ & $\begin{array}{l}\text { Nystatin, rutosides, immuno-globulines, } \\
\text { panthenol } \pm \text { PVP-iodine }\end{array}$ & Significant reduction \\
\hline $\mathrm{CT}+\mathrm{RT}$ & Hasenau et al. (248) & No/yes/no & $P$ & Hydrogen peroxide, PVP-iodine, dexpanthenol, nystatin & $\begin{array}{l}\text { Lower incidence and severity of oral } \\
\text { mucositis }\end{array}$ \\
\hline $\mathrm{RT}$ & Spijkervet et al. (227) & No/yes/no & $P$ & $\begin{array}{l}\text { Lozenges of polymyxin, tobramycin, amphotericin vs. } \\
\text { historical controls }\end{array}$ & Lower incidence of mucositis \\
\hline $\mathrm{RT}$ & Mattews et al. (228) & Yes/yes/no & $P$ & $\begin{array}{l}\text { Sucralfate + (ciprofloxacin or ampicillin) + clotrimazole } \\
\text { vs. sucralfate }\end{array}$ & $\begin{array}{l}\text { Significant reduction of incidence and } \\
\text { severity }\end{array}$ \\
\hline $\mathrm{RT}$ & Symonds et al. (249) & Yes/yes/yes & $P$ & $\begin{array}{l}\text { Pastilles containing polymyxin, tobramycin, } \\
\text { amphothericin vs. placebo }\end{array}$ & Significant reduction of severe mucositis \\
\hline $\mathrm{RT}$ & Okuno et al. (250) & Yes/yes/yes & $P+T$ & $\begin{array}{l}\text { Lozenges of polymyxin, tobramycin, amphotericin vs. } \\
\text { placebo }\end{array}$ & $\begin{array}{l}\text { Significant reduction of oral discomfort, no } \\
\text { objective difference }\end{array}$ \\
\hline $\mathrm{RT}$ & Okuno et al. (250) & Yes/yes/no & $\mathrm{T}$ & $\begin{array}{l}\text { Amphotericin + colistin + tobramycin + chlorhexidine vs. } \\
\text { placebo }\end{array}$ & Decreased oral discomfort \\
\hline RT & Symonds et al. (249) & Yes/yes/yes & $P$ & Amphotericin + tobramycin + polymyxin vs. placebo & $\begin{array}{l}\text { Significant reduction of the incidence of } \\
\text { sever mucositis }\end{array}$ \\
\hline $\mathrm{RT}$ & Spijkervet et al. (227) & No/yes/no & $P$ & $\begin{array}{l}\text { Amphotericin }+ \text { tobramycin }+ \text { polymyxin vs. historical } \\
\text { chlorhexidine or placebo group }\end{array}$ & $\begin{array}{l}\text { Significant reduction of severity of } \\
\text { mucositis }\end{array}$ \\
\hline RT & Carl et al. (251) & No/yes/no & $\mathrm{P}+\mathrm{T}$ & Chamomile vs. historical group & Low incidence of mucositis \\
\hline $\mathrm{RT}$ & Fidler et al. (126) & Yes/yes/yes & $P$ & Chamomile vs. placebo, cryoprophylaxis in all patients & No difference \\
\hline RT & Abdelaal et al. (252) & No/no/no & $P$ & High-dose betamethasone & $\begin{array}{l}\text { Impressive prevention of mucositis } \\
\text { incidence }\end{array}$ \\
\hline RT & Kim et al. (253) & Yes/yes/yes & $P+T$ & Benzydamine vs. placebo & Significant reduction (less pain) \\
\hline
\end{tabular}


TABLE 10 | Continued

\begin{tabular}{|c|c|c|c|c|c|}
\hline Injury & Reference & $\begin{array}{l}\text { Randomized/ } \\
\text { controlled/ } \\
\text { double blind }\end{array}$ & $\mathbf{P} / \mathbf{T}$ & Application/doses & Results \\
\hline RT & Epstein et al. (156) & Yes/yes/yes & $P+T$ & Benzydamine vs. placebo & $\begin{array}{l}\text { Significant reduction of incidence and } \\
\text { severity }\end{array}$ \\
\hline $\mathrm{RT}$ & $\begin{array}{l}\text { Samaranayake } \\
\text { et al. (254) }\end{array}$ & Yes/no/no & $\mathrm{P}$ & Benzydamine vs. chlorhexidine & No difference (more discomfort) \\
\hline $\mathrm{CT}+\mathrm{RT}$ & Prada et al. (255) & Yes/yes/yes & $P+T$ & Benzydamine vs. placebo & Significant reduction \\
\hline $\mathrm{RT}$ & Huang et al. (73) & Yes/yes/yes & $\mathrm{P}$ & Parenteral glutamine vs. placebo & No difference \\
\hline $\mathrm{CT}+\mathrm{RT}$ & Porteder et al. (256) & No/yes/no & $\mathrm{P}$ & PGE2 or nothing & Significant reduction (less pain) \\
\hline $\mathrm{RT}$ & Matejka et al. (171) & No/yes/no & $\mathrm{T}$ & PGE2 tablets four times a day & Reduction of mucositis severity \\
\hline $\mathrm{CT}+\mathrm{RT}$ & Hasenau et al. (248) & No/no/no & $P+T$ & $\mathrm{P}+\mathrm{T}$ hydrogen peroxide, nystatin & Lower incidence of mucositis \\
\hline $\mathrm{RT}$ & Rothwell et al. (109) & Yes/yes/yes & $P$ & $\begin{array}{l}\text { Hydrocortisone, nystatin, tetracyclines, diphenhydramine } \\
\text { vs. placebo }\end{array}$ & Significant reduction of incidence \\
\hline $\mathrm{RT}$ & $\begin{array}{l}\text { Maciejewski } \\
\text { et al. (257) }\end{array}$ & No/yes/no & $\mathrm{P}$ & Applied to one side of buccal mucosa & $\begin{array}{l}\text { Significant reduction compared with } \\
\text { contralateral side }\end{array}$ \\
\hline $\mathrm{RT}$ & Barker et al. (100) & Yes/yes/yes & & $\begin{array}{l}\text { Oral hygiene + sucralfate vs. } \\
\text { diphenhydramine + kaolin-pectin }\end{array}$ & No difference \\
\hline $\mathrm{CT}+\mathrm{RT}$ & Berger et al. (161) & No/yes/no & $\mathrm{T}$ & Capsaicin in a candy vehicle & Significant temporary pain relief \\
\hline $\mathrm{CT}+\mathrm{RT}$ & Mills (197) & Yes/yes/no & $\mathrm{P}$ & Beta-carotene or nothing & Decreased severity in the treatment group \\
\hline $\mathrm{RT}$ & Bourhis et al. (258) & Yes/yes/no & $\mathrm{P}$ & Amifostine or nothing & $\begin{array}{l}\text { Marked reduction of mucositis (tolerance } \\
\text { was poor) }\end{array}$ \\
\hline RT & $\begin{array}{l}\text { Koukourakis } \\
\text { et al. ( } 259)\end{array}$ & Yes/yes/yes & $P$ & Amifostine vs. saline & Significant reduction of mucositis \\
\hline $\mathrm{RT}$ & Schonekas et al. (260) & No/yes/no & $\mathrm{P}$ & Amifostine vs. controls & Significant reduction of mucositis \\
\hline $\mathrm{RT}$ & Wagner et al. (261) & Yes/yes/no & $\mathrm{P}$ & Amifostine or nothing & Significant reduction of mucositis \\
\hline $\mathrm{CT}+\mathrm{RT}$ & Buntzel et al. (262) & Yes/yes/no & $\mathrm{P}$ & Amifostine or nothing & $\begin{array}{l}\text { Significant reduction of mucositis and } \\
\text { xerostomia }\end{array}$ \\
\hline $\mathrm{CT}+\mathrm{RT}$ & Peters et al. (263) & Yes/yes/no & $\mathrm{P}$ & Amifostine or nothing & No significant difference \\
\hline $\mathrm{CT}+\mathrm{RT}$ & Vacha et al. (264) & Yes/yes/no & $\mathrm{P}$ & Amifostine or nothing & Trend toward reduction of mucositis \\
\hline $\mathrm{CT}+\mathrm{RT}$ & Osaki et al. (199) & Yes/yes/no & $\mathrm{P}$ & Vitamins $\mathrm{C}+\mathrm{E}$, glutathione \pm azelastine & Significant reduction \\
\hline $\mathrm{RT}$ & Pillsbury et al. (165) & Yes/yes/yes & $\mathrm{P}$ & Indomethacin vs. placebo & Significant delay of mucositis onset \\
\hline $\mathrm{CT}+\mathrm{RT}$ & Mose et al. (201) & No/yes/no & $P$ & i.m. immunoglobulins & $\begin{array}{l}\text { Significant reduction in } \mathrm{CT}+\mathrm{RT} \text { patients, } \\
\text { no difference in } \mathrm{RT}\end{array}$ \\
\hline $\mathrm{RT}$ & Wagner et al. (265) & Yes/yes/no & $\mathrm{P}$ & $\mathrm{RT}+\mathrm{GM}-\mathrm{CSF}$ vs. historical control & Significant lower severity of mucositis \\
\hline $\mathrm{RT}$ & Makkonen et al. (140) & No/yes/no & $\mathrm{P}$ & Sucralfate \pm GM-CSF & No difference \\
\hline $\mathrm{RT}$ & Kannan et al. (193) & No/yes/no & $\mathrm{P}$ & $\mathrm{RT}+\mathrm{GM}-\mathrm{CSF}$ & Lower incidence of severe mucositis \\
\hline $\mathrm{CT}+\mathrm{RT}$ & Rosso et al. (266) & No/yes/no & $P$ & $\begin{array}{l}\text { GM-CSF vs. historical control sig. lower incidence of } \\
\text { severe mucositis }\end{array}$ & Lower incidence of severe mucositis \\
\hline $\mathrm{RT}$ & Mascarin et al. (267) & Yes/yes/no & $\mathrm{P}$ & $\mathrm{RT} \pm \mathrm{G}-\mathrm{CSF}$ & Less treatment interruptions only \\
\hline RT & Schneider et al. (268) & Yes/yes/yes & $P$ & $\mathrm{RT} \pm \mathrm{G}-\mathrm{CSF}$ & $\begin{array}{l}\text { Significant reduced incidence of severe } \\
\text { mucositis }\end{array}$ \\
\hline $\mathrm{CT}+\mathrm{RT}$ & Bubley et al. (236) & Yes/yes/yes & $P$ & Acyclovir vs. placebo & $\begin{array}{l}\text { No impact upon incidence and severity of } \\
\text { mucositis }\end{array}$ \\
\hline
\end{tabular}

RT, radiotherapy; P/T, prevention or treatment; CT, chemotherapy; HD-CT, high-dose chemotherapy; BMT, bone marrow transplantation; TBI, total body irradiation; i.m., intramuscular; G-CSF, colony-stimulating factor; GM-CSF, granulocyte-macrophage colony-stimulating factor.

${ }^{a}$ Published with permission from Dr. Christoph C. Zielinski.

lidocaine and holding $5 \mathrm{~mL}$ of it in mouth for $1 \mathrm{~min}$ then spitting it out before meals have been shown to be helpful for better alimentation (101). One clinical trial showed dyclonine hydrochloride to have a superior effect among all other agents without significant difference recorded (102). The most effective anesthetic agent is still to be determined. Benzocaine gel is another locally applied bioadhesive agent containing benzyl alcohol (10\%) and is 
used to relieve pain and facilitate eating and drinking in mild and moderate RIOM (103). Benzocaine-containing lozenges are diluted to alleviate the pain sensation and mechanical sensitivity in mild to moderate OM $(104,105)$. The "magic mouthwash" (lidocaine, diphenhydramine, magnesium aluminum hydroxide) and morphine mouth washes are preferable and have been reported by patients to be effective in alleviating pain in RIOM (106-108).

5. The application of corticosteroids mouthwashes has shown promising results. The limited availability of a large-scale data is a gap that should be bridged through relevant clinical studies (109).

6. Allopurinol and uridine were shown to be effective in reducing 5-fluorouracil oral toxicity in preclinical studies (110-114). Despite these results, they were ineffective approaches in randomized clinical trials as a therapy to reduce the treatment-related oral toxicity $(115,116)$.

7. Chlorhexidine is a bisguanidine exhibiting broadspectrum antibacterial and antimycotic activities. The clinical trials done with chlorhexidine concluded that it cannot be recommended for the prophylaxis or the treatment of RIOM (117-120). Alcohol-containing chlorhexidine mouth rinse should be avoided during clinical oral ulceration. Therefore, the MASCC/ISOO guidelines recommend against the use of chlorhexidine mouth rinse for prevention or treatment OM (33).

8. Artificial saliva spray is an over-the-counter agent frequently used to alleviate mucosal dryness in mild cases of RIOM (121).

9. Chamomile has anti-inflammatory, antipeptic, antispasmodic, and antibacterial effects. It was investigated with encouraging results as an emulsion therapy for CT-induced mucositis (122-126). Studies are needed for its application in RIOM to determine its efficacy.

10. Honey has been investigated in many preclinical studies due to its mucosal protective effect that was confirmed as a reduction in the incidence and severity of RIOM (127-132). However, the available clinical trial used only Manuka honey, and it appears to contradict the preclinical studies' results (133). More studies are needed to confirm the therapeutic potential of honey in RIOM.

11. Sucralfate is a basic aluminum salt of sucrose sulfate that was used as mouthwash to reduce the intensity of RIOM and CCRT-induced mucositis as well (39, 100, 134-151). Despite its long application history, it is considered to have little effect in RIOM when compared to oral hygiene and symptomatic mucositis therapy (2). MASCC/ISOO Mucositis Guidelines did not find enough evidence for the beneficial application of sucralfate in OM (34).

12. Vitamin A and its derivatives have anti-inflammatory and epithelial proliferative effect (152). Topical tretinoin has been shown to reduce the oral complications during bone marrow (BM) transplantation (153).

13. Vitamin-E (tocopherol) has been shown to lower the oxidative damage of the oral mucosa and reduce the incidence of symptomatic RIOM in head and neck cancer patients in a randomized double-blind clinical trial $(152,154)$.

14. Sodium alginate was shown to reduce the discomfort and the severity of RIOM in a randomized clinical trial (155).

15. Benzydamine hydrochloride is a non-steroidal antimicrobial, anti-inflammatory, anesthetic, and analgesic agent that reduces pro-inflammatory cytokine production, scavenges the ROS, and acts as membrane stabilization and as an antimicrobial agent $(118,156,157)$. When compared with chlorhexidine, patients with RIOM treated with benzydamine hydrochloride found more discomfort (118). Benzydamine hydrochloride failed to be approved by the US-FDA for OM management. Because of a negative interim analysis, a recent phase III trial for benzydamine hydrochloride therapy in RIOM was stopped (33).

16. Povidone-iodine is an antiviral, antibacterial, and antifungal agent. Randomized clinical study showed that povidone-iodine reduces the incidence, severity, and duration of CCRT-induced OM, in addition to its advantages of being cheap and easily applied $(118,158-160)$.

17. Capsaicin is an inhibitor of neutrophils that reduces the pain sensation. One clinical trial showed that orally applied capsaicin caused temporary relief of pain in mucositis caused by RT and CT (161). However, more studies are needed for optimization of its analgesic effect.

II Systemically applied agents

1. Cyclooxegenase-2 inhibitors that have different mechanisms of action were applied in the management of RIOM. They suppress NF- $\kappa \mathrm{B}$, reduce pro-inflammatorycytokine production, and inhibit angiogenesis (162-164). A randomized placebo-controlled trial showed that prophylactic systemic administration of indomethacin, a COX-2 inhibitor, significantly lowered the severity and delayed the onset of RIOM (165). In addition, PG $\mathrm{E} 1$ and $\mathrm{E} 2$ showed improvement in OM induced by RT and CT in few studies; however, their application is still controversial (166-172).

2. $N$-acetylcysteine is an antioxidant that has been shown to suppress NF- $\kappa \mathrm{B}$ activation $(173,174)$. Because of its proven radioprotective role in RT-induced dermatitis, bone injury, liver toxicity, and intestinal injury (173188), $\mathrm{N}$-acetylcysteine was recommended as a candidate for a trial in RIOM. In a placebo-controlled phase II trial of patients with head and neck cancer, $N$-acetylcysteine significantly reduced the severity of RIOM (33).

3. Colony-stimulating factor and granulocyte-macrophage colony-stimulating factor (GM-CSF) systemic therapy recruit neutrophils to the tissue injury site (189). Local application of GM-CSF mouthwash was shown marked alleviation of RIOM in several studies (2). Nevertheless, in clinical trials, its systemic application therapeutic value appears controversial $(190,191)$. SC GM-CSF reduced the severity of $\mathrm{OM}$ in patients treated with accelerated RT (192). In another randomized clinical study, systemic GM-CSF reduced the incidence of RIOM; however, 


\begin{tabular}{|c|c|c|c|}
\hline NCT number & Title & Conditions & Last updated \\
\hline NCT02508389 & $\begin{array}{l}\text { A Study of GC4419 Protection against Radiation Induced Oral Mucositis in Patients with } \\
\text { Head \& Neck Cancer }\end{array}$ & Radiation-Induced Oral Mucositis & 23 November 2015 \\
\hline NCT00698204 & Cox-2 Inhibition in Radiation-Induced Oral Mucositis & Oral Mucositis & 7 May 2014 \\
\hline NCT00814359 & $\begin{array}{l}\text { Magic Mouthwash Plus Sucralfate Versus Benzydamine Hydrochloride for the Treatment of } \\
\text { Radiation-Induced Mucositis }\end{array}$ & Head and Neck Cancer|Mucositis & 19 January 2011 \\
\hline NCT01400620 & $\begin{array}{l}\text { Safety and Efficacy of IZN-6N4 Oral Rinse for the Prevention of Oral Mucositis in Patients with } \\
\text { Head and Neck Cancer }\end{array}$ & Oral Mucositis & 9 November 2015 \\
\hline NCT00051441 & $\begin{array}{l}\text { Safety \& Efficacy Study of Benzydamine Oral Rinse for the Treatment of Oral Mucositis (Mouth Sores) } \\
\text { Resulting From Radiation Therapy for Cancer of the Oral Cavity, Oropharynx, or Nasopharynx }\end{array}$ & Stomatitis|Radiation Effects & 17 May 2011 \\
\hline NCT02608879 & $\begin{array}{l}\text { Oral Care Protocol for the Management of Chemotherapy and Radiation Therapy-Induced Oral } \\
\text { Mucositis }\end{array}$ & Oral Mucositis|Oral Cancer & 17 November 2015 \\
\hline NCT01465308 & The Effect of Honey on Xerostomia and Oral Mucositis & Head and Neck Cancer & 7 October 2014 \\
\hline NCT01375088 & $\begin{array}{l}\text { Assessing the Preventing and Therapeutic Effect of Propolis in Radiotherapy Induced Mucositis } \\
\text { of Head and Neck Cancers }\end{array}$ & Radiation-induced Mucositis of Oral Mucous Membranes & 21 November 2012 \\
\hline NCT01066741 & $\begin{array}{l}\text { Prevention of Radiation-induced Severe Oral Mucositis in Oral Cavity, Oropharynx, Hypopharynx, } \\
\text { and Cavum Cancer }\end{array}$ & Oropharynx Cancer|Hypopharynx Cancer & 31 October 2012 \\
\hline NCT00006994 & $\begin{array}{l}\text { S9908: Glutamine in Treating Mucositis Caused by Radiation Therapy in Patients with Newly } \\
\text { Diagnosed Cancer of the Mouth or Throat }\end{array}$ & Cancer-related Problem/Condition|Head and Neck Cancer|Pain & 17 November 2015 \\
\hline NCT02430298 & $\begin{array}{l}\text { Topical/Oral Melatonin for Preventing Concurrent Radiochemotherapy Induced Oral Mucositis/ } \\
\text { Xerostomia Cancer Patients }\end{array}$ & Head and Neck Cancer & 12 May 2015 \\
\hline NCT02397486 & $\begin{array}{l}\text { The Impact of Pentoxifylline and Vitamin E on Radiotherapy-induced Toxicity in Head \& Neck } \\
\text { Cancer Patients }\end{array}$ & Head and Neck Neoplasms & 27 May 2015 \\
\hline NCT01941992 & $\begin{array}{l}\text { Role of SAMITAL }{ }^{\otimes} \text { in the Relief of Chemoradiation (CT-RT) Induced Oral Mucositis in Head and } \\
\text { Neck Cancer Patients }\end{array}$ & Head-and-neck Squamous Cell Carcinoma|Oral Mucositis & 24 March 2015 \\
\hline NCT01318889 & Dexpanthenol Mouthwash to Treat Oral Mucositis & Oral Mucositis (Ulcerative) Due to Radiation & 5 July 2011 \\
\hline NCT02016807 & $\begin{array}{l}\text { ZeroTolerance Mucositis: Managing Oral and Alimentary Mucositis with High Potency } \\
\text { Sucralfate-ProThelial }\end{array}$ & Oral Mucositis|Nausea|Vomiting|Diarrhea & 16 December 2013 \\
\hline NCT00293462 & $\begin{array}{l}\text { GM-CSF Mouthwash for Preventing and Treating Mucositis in Patients Who Are Undergoing } \\
\text { Radiation Therapy for Head and Neck Cancer }\end{array}$ & Head and Neck Cancer|Mucositis|Radiation Toxicity & 14 May 2013 \\
\hline NCT00728585 & $\begin{array}{l}\text { Palifermin in Preventing Oral Mucositis Caused by Chemotherapy and/or Radiation Therapy in } \\
\text { Young Patients Undergoing Stem Cell Transplant }\end{array}$ & $\begin{array}{l}\text { Breast Cancer|Graft vs. Host Disease|Kidney } \\
\text { Cancer|Leukemia|Lymphoma|Mucositis|Multiple Myeloma|Plasma } \\
\text { Cell Neoplasm|Myelodysplastic Syndromes|Neuroblastoma|Ovarian } \\
\text { Cancer|Sarcoma|Testicular Germ Cell Tumor }\end{array}$ & 30 May 2013 \\
\hline NCT02604329 & Feasibility Study of a Protocol to Treat Pediatric Oral Mucositis by Low-Level Laser Therapy & Oral Mucositis & 12 November 2015 \\
\hline NCT02075749 & Comparing Triamcinolone Acetonide Mucoadhesive Films with Licorice Mucoadhesive Films & Mucositis & 9 July 2014 \\
\hline NCT01385748 & Efficacy and Safety Study of Clonidine Lauriad ${ }^{\circledast}$ to Treat Oral Mucositis & Oral Mucositis & 7 July 2015 \\
\hline NCT01707641 & $\begin{array}{l}\text { Effect of Lactobacillus Brevis CD2 in Prevention of Radio-chemotherapy Induced Oral Mucositis in } \\
\text { Head and Neck Cancer }\end{array}$ & Mucositis & 19 May 2014 \\
\hline
\end{tabular}


TABLE 11 | Continued

\begin{tabular}{|c|c|c|c|}
\hline NCT number & Title & Conditions & Last updated \\
\hline NCT00613743 & $\begin{array}{l}\text { Effect of Topical Morphine (Mouthwash) on Oral Pain Due to Chemo- and/or Radiotherapy Induced } \\
\text { Mucositis }\end{array}$ & Cancer|Mucositis & 12 January 2010 \\
\hline NCT00431925 & Can Cytokines Predict the Severity of Acute Mucositis and the Need for Gastrostomy Tubes (PEG)? & Oral Mucositis|Xerostomia|Weight Loss|Head and Neck Cancer & 9 August 2007 \\
\hline NCT01806272 & $\begin{array}{l}\text { Recombinant Human Granulocyte Macrophage Colony-Stimulating Factor (rhGM-CSF) Treating Oral } \\
\text { Mucositis }\end{array}$ & Nasopharyngeal Cancers & 27 March 2013 \\
\hline NCT01876407 & $\begin{array}{l}\text { Effectiveness of Low Energy Laser Treatment in Oral Mucositis Induced by Chemotherapy and } \\
\text { Radiotherapy in Head and Neck Cancer }\end{array}$ & Oral Mucositis & 30 April 15 \\
\hline NCT00584597 & A Trial of Homeopathic Medication TRAUMEEL S for the Treatment of Radiation-Induced Mucositis & Mucositis|Head and Neck Cancer & 10 December 2010 \\
\hline NCT00615420 & $\begin{array}{l}\text { A Randomized Placebo-Controlled Trial of Manuka Honey for Oral Mucositis Due to Radiation } \\
\text { Therapy for Cancer }\end{array}$ & Radiotherapy-Induced Mucositis|Head and Neck Cancer & 22 May 2012 \\
\hline NCT01898091 & Herbal Mouthrinse for Oral Mucositis Study & Oral Mucositis & 21 Septmebr 2015 \\
\hline NCT01772706 & $\begin{array}{l}\text { Laser Mucite ORL: Effectiveness of Laser Therapy for Mucositis Induced by a Radio-chemotherapy in } \\
\text { Head and Neck Cancer }\end{array}$ & $\begin{array}{l}\text { Oral Squamous Cell Carcinoma|Squamous Cell Carcinoma of } \\
\text { Oropharynx|Squamous Cell Carcinoma of Hypopharynx|Oral } \\
\text { Mucositis }\end{array}$ & 17 January 2013 \\
\hline NCT01837446 & Morphine Mouthwash for Management of Oral Mucositis in Patients with Head and Neck Cancer & Stomatitis & 22 April 2013 \\
\hline NCT02309437 & $\begin{array}{l}\text { Early Use of Opioid to Control Local Mucosa Pain Induced by Irradiation in Nasopharyngeal } \\
\text { Carcinoma Patients }\end{array}$ & Nutrition Disorders|Quality of Life & 3 December 2014 \\
\hline NCT01668849 & $\begin{array}{l}\text { Edible Plant Exosome Ability to Prevent Oral Mucositis Associated with Chemoradiation Treatment } \\
\text { of Head and Neck Cancer }\end{array}$ & Head and Neck Cancer|Oral Mucositis & 12 May 2015 \\
\hline NCT01975688 & A Pharmacokinetic Study of Single Doses of Sativex in Treatment-Induced Mucositis & Head and Neck Squamous Cell Carcinoma & 12 May 2015 \\
\hline NCT01252498 & Evaluation of the Role of Prostaglandins in Radiation-induced Mucositis & Cancer of the Head and Neck|Radiotherapy & 3 February 2014 \\
\hline NCT01840436 & $\begin{array}{l}\text { Efficacy of MUCIPLIQ on the Incidence of Radio-chemotherapy-Induced Mucositis in Patients } \\
\text { Suffering From Oral Cancer }\end{array}$ & Oral Mucositis|Carcinoma in Situ of Upper Respiratory Tract & 15 May 2014 \\
\hline NCT00699569 & Hyperimmune Colostrum and Oral Mucositis & Head and Neck Cancer & 22 July 2008 \\
\hline NCT02555501 & Oral Mucositis and Laser Therapy Associated with Photodynamic Therapy & Oral Mucositis & 18 September 2015 \\
\hline NCT02050503 & $\begin{array}{l}\text { Intranasal Transmucosal Fentanyl Pectin for Breakthrough Cancer Pain in Radiation-Induced } \\
\text { Oropharyngeal Mucositis }\end{array}$ & $\begin{array}{l}\text { Breakthrough Pain|Mucositis|Radiotherapy|Chemotherapy|Head and } \\
\text { Neck Cancer }\end{array}$ & 16 March 2015 \\
\hline NCT01883908 & $\begin{array}{l}\text { Acupuncture in Reducing the Severity of Chemoradiation-Induced Mucositis in Patients with } \\
\text { Oropharyngeal Cancer }\end{array}$ & Mucositis|Oropharyngeal Cancer & 3 September 2015 \\
\hline NCT01432873 & Oral Selenium Therapy for the Prevention of Mucositis & Mucositis|Hematopoietic Stem Cell transplantation & 31 May 2012 \\
\hline
\end{tabular}


another study did not show the same result $(140,193)$. Systemic GM-CSF therapeutic potential is still controversial and requires further investigation.

4. Transforming growth factor- $\beta 3$ inhibits the oral basal cell proliferation. It was shown to reduce the incidence of CT-induced mucositis (194). However, a reliable clinical trial is needed to assess its therapeutic potential with RT.

5. Beta-carotene's antioxidative effect $(195,196)$ was implemented in a randomized clinical trial where there was a significant reduction in the incidence of severe OM in CCRT (197).

6. Analgesics are strong candidates for alleviating the pain related to RIOM. A retrospective study showed that opioid therapy remains a corner stone for OM pain management in CCRT, as suggested by the MASCC/ ISOO guidelines $(33,198)$.

7. Azelastine is a potent second-generation selective histamine antagonist that is used as an anti-inflammatory and antioxidant agent. One clinical trial showed significant reduction in the incidence and the severity of OM with CCRT (199).

8. Propantheline is an anticholinergic agent that reduces the salivary flow. One clinical trial showed that propantheline and oral cryotherapy may be feasible and effective in reducing mucosal toxicity in cancer patients receiving high-dose CT (200). However, studies are needed for RIOM.

9. Immunoglobulins have lower salivary and systemic levels in patient receiving antineoplastic therapy. They have immune-modulating and anti-inflammatory properties. Intravenous or intramuscular immunoglobulins are frequently applied as prophylactic and therapeutic options for RIOM $(158,201)$.

10. Systemic corticosteroids were used in RIOM management. A double-blind placebo-controlled randomized trial has shown a tendency toward reduced RT interruption in prednisone-treated relative to placebo-treated patient groups without evidence of reduced RIOM incidence or severity (202).

11. Pentoxifylline regulates endotoxin-induced production of TNF- $\alpha$. Although the preclinical studies showed significant reduction in the severity of RIOM with pentoxifylline (203), the clinical trials show that it is not effective in reducing the antineoplastic oral toxicity (204-208).

12. Salicylic acid derivatives should be avoided due to the increased risk for bleeding (34-36).

13. Sphingomyelinase and ceramide synthase inhibitors can be a potential candidate for RIOM. They inhibit the ceramide pathway-mediated RT-induced apoptosis (209-216). No current clinical trials have been started for them yet.

III Oral microbial load reduction agents

1. Antimicrobial agents showed beneficial effect in prophylaxis and reduction of the severity of RIOM. RT injury leads to a change in the mucosal membrane barrier, salivary flow, and composition which favor the growth and colonization of different bacterial species, mainly Gram-negative bacteria. Many preclinical studies have investigated the therapeutic effect of different antimicrobial agents in RIOM (217-220). The FDA has granted fast track designation for brilacidin-OM, an oral rinse formulation of defensin-mimetic brilacidin (221-223), for the prevention of OM. There is a current phase II clinical trial to evaluate the safety and efficacy of brilacidin oral rinse in patients with head and neck cancer (NCT02324335).

2. Fungal infections are not involved directly in the development in RIOM, rather they can complicate the situation, especially in immunocompromised patients, and that is why the use of antifungal agents have been applied in RIOM treatment. A clinical study has shown that systemic fluconazole prophylaxis caused a significant beneficial effect on the severity of OM and on radiotherapy interruptions (224). The same effect was noted in randomized clinical trials investigating clotrimazole (2). Some oral mouthwashes containing amphotericin B have shown similar effects; however, due to carrier allergy, there might be a limitation in its application (225).

3. Antibacterial agents have been investigated in mucositis depending on a hypothesis stating that aerobic species (e.g., Pseudomonas spp. and Staphylococcus epidermidis), anaerobic bacteria (e.g., Bacteroides spp., and Veillonella spp.), and endotoxin of aerobic Gram-negative bacilli are considered a main contributor in the development of the secondary infection phase in $\operatorname{RIOM}(2,226)$. Antibiotic lozenges with polymyxin-E and tobramycin have protected against severe mucositis when compared to placebo or chlorhexidine (227). In addition, ciprofloxacin- and ampicillin-containing mouthwashes showed similar effect $(228,229)$.

4. Antiviral agents against herpes simplex virus (HSV) type I and varicella zoster virus (VZV) were applied topically and systematically. HSV and VZV are the most common viral infections that aggravate RIOM in seropositive and myelo-suppressed patients (230-232). Systemic and topical acyclovir was investigated and applied in RIOM management and caused a reduction in the oral herpetic infections without an evident prophylactic role against OM itself (233-238).

\section{CELLULAR THERAPIES FOR RIOM}

Bone marrow-derived mesenchymal stromal cells (bmMSCs) therapy have been applied in fractionated radiation-induced OM where the administration of a systemic single dose of six million MSCs resulted in a significant decrease in $\mathrm{ED}_{50}$ (the RT dose that produces ulcer in $50 \%$ of irradiated mice) (239). The first MSCs therapy for RIOM was done in 2014 by Schmidt et al. (239). They concluded that transplantation of BM or bmMSCs could modulate RIOM in fractionated RT, depending on the time of transplantation (239). Nevertheless, in another study, the authors concluded that bmMSCs transplantation had no therapeutic benefits on RIOM in single-dose RT when compared to the therapeutic effect of mobilization of endogenous BM stem 
cells (240). More studies are needed in this field building on the initial studies, which showed significant and clinically relevant therapeutic gain of MSCs therapy for RIOM (Table 10).

\section{Clinical Trials for RIOM}

Table 10 summarizes the clinical trials that were done until 2001 for prevention (P) and treatment (T) of RIOM (2). The current clinical trials for RIOM are summarized in Table $\mathbf{1 1}$ and were found when searching the clinical trials website of the National Institute of Health for RIOM. We have documented 40 RIOM treatment and prevention clinical trials.

\section{CONCLUSION}

Despite its high incidence, RIOM is a self-limited radiotherapyinduced normal tissue injury. It is a dose-limiting toxicity in most cases of head and neck cancer patients. However, in moderately to severely sick patients, it could be a lethal injury. Many preclinical and clinical studies have been conducted for the prevention and treatment of RIOM. Currently, there are numerous prevention and treatment strategies for RIOM. However, there is no single agent or management regimen that has been agreed upon between caregivers that significantly improves RIOM to a clinically relevant and satisfactory standard.

\section{REFERENCES}

1. Muanza TM, Cotrim AP, McAuliffe M, Sowers AL, Baum BJ, Cook JA, et al. Evaluation of radiation-induced oral mucositis by optical coherence tomography. Clin Cancer Res (2005) 11(14):5121-7. doi:10.1158/1078-0432. CCR-05-0403

2. Köstler WJ, Hejna M, Wenzel C, Zielinski CC. Oral mucositis complicating chemotherapy and/or radiotherapy: options for prevention and treatment. CA Cancer J Clin (2001) 51(5):290-315. doi:10.3322/ canjclin.51.5.290

3. Al-Ansari S, Zecha JAEM, Barasch A, de Lange J, Rozema FR, RaberDurlacher JE. Oral mucositis induced by anticancer therapies. Curr Oral Health Rep (2015) 2:202-11. doi:10.1007/s40496-015-0069-4

4. Karthaus M, Rosenthal C, Ganser A. Prophylaxis and treatment of chemo- and radiotherapy-induced oral mucositis - are there new strategies? Bone Marrow Transplant (1999) 24(10):1095-108. doi:10.1038/ sj.bmt. 1702024

5. Naidu MU, Ramana GV, Rani PU, Mohan IK, Suman A, Roy P. Chemotherapy-induced and/or radiation therapy-induced oral mucositiscomplicating the treatment of cancer. Neoplasia (2004) 6(5):423-31. doi:10.1593/neo.04169

6. Rosenthal C, Karthaus M. [Current approaches in prevention and therapy of chemo- and radiotherapy-induced oral mucositis]. Wien Med Wochenschr (2001) 151(3-4):53-65.

7. Volpato LE, Silva TC, Oliveira TM, Sakai VT, Machado MA. Radiation therapy and chemotherapy-induced oral mucositis. Braz J Otorhinolaryngol (2007) 73(4):562-8. doi:10.1016/S1808-8694(15)30110-5

8. Sonis ST. Mucositis as a biological process: a new hypothesis for the development of chemotherapy-induced stomatotoxicity. Oral Oncol (1998) 34(1):39-43. doi:10.1016/S1368-8375(97)00053-5

9. Feller L, Essop R, Wood NH, Khammissa RA, Chikte UM, Meyerov R, et al. Chemotherapy- and radiotherapy-induced oral mucositis: pathobiology, epidemiology and management. SADJ (2010) 65(8):372-4.

10. Sonis ST. Pathobiology of oral mucositis: novel insights and opportunities. J Support Oncol (2004) 5:3-11.

11. Redding SW. Cancer therapy-related oral mucositis. J Dent Educ (2005) 69(8):919-29.
Nevertheless, the current guidelines recommend good oral care, IMRT, radiation shields, palifermin, amifostine, and cryotherapy for RIOM prevention. RIOM treatment focuses on palliative measures and symptoms relief; e.g., pain management, nutritional support, good oral hygiene, and reduced oral microbial load. Interestingly, mesenchymal stromal cells therapy for RIOM shows promise for potential therapeutic and clinically relevant benefits. However, more studies are still needed to confirm such therapeutic potential.

\section{AUTHOR CONTRIBUTIONS}

OM: conception and design, collection and/or assembly of data, review writing, and final approval of the review. NE: conception, design, and final approval of the review. TM: conception and design, financial support, and final approval of the review.

\section{FUNDING}

$\mathrm{OM}$ is an awardee of the Lady Davis Institute/Toronto-Dominion Bank studentship. This study was supported partially by Ride To Conquer Cancer (RTCC, Jewish General Hospital Foundation) and Fonds de Recherche du Quebec-Santé (FRQS) grants. English language editing was done by Jenny Warrington.

12. Elting LS, Cooksley CD, Chambers MS, Garden AS. Risk, outcomes, and costs of radiation-induced oral mucositis among patients with head-andneck malignancies. Int J Radiat Oncol Biol Phys (2007) 68(4):1110-20. doi:10.1016/j.ijrobp.2007.01.053

13. Luo DH, Hong MH, Guo L, Cao KJ, Deng MQ, Mo HY. [Analysis of oral mucositis risk factors during radiotherapy for nasopharyngeal carcinoma patients and establishment of a discriminant model]. Ai Zheng (2005) 24(7):850-4.

14. Chen SC, Lai YH, Huang BS, Lin CY, Fan KH, Chang JT. Changes and predictors of radiation-induced oral mucositis in patients with oral cavity cancer during active treatment. Eur J Oncol Nurs (2015) 19(3):214-9. doi:10.1016/j. ejon.2014.12.001

15. Eilers J, Million R. Prevention and management of oral mucositis in patients with cancer. Semin Oncol Nurs (2007) 23:201-12. doi:10.1016/j. soncn.2007.05.005

16. Sonis ST. Mucositis: the impact, biology and therapeutic opportunities of oral mucositis. Oral Oncol (2009) 45(12):1015-20. doi:10.1016/j.oraloncology. 2009.08.006

17. Sonis ST, Elting LS, Keefe D, Peterson DE, Schubert M, Hauer-Jensen M, et al. Perspectives on cancer therapy-induced mucosal injury. Cancer (2004) 100(9 Suppl):1995-2025. doi:10.1002/cncr.20162

18. Chamberlain G, Fox J, Ashton B, Middleton J. Concise review: mesenchymal stem cells: their phenotype, differentiation capacity, immunological features, and potential for homing. Stem Cells (2007) 25(11):2739-49. doi:10.1634/ stemcells.2007-0197

19. Sonis ST. The pathobiology of mucositis. Nat Rev Cancer (2004) 4:277-84

20. Scully C, Epstein J, Sonis S. Oral mucositis: a challenging complication of radiotherapy, chemotherapy, and radiochemotherapy. Part 2: diagnosis and management of mucositis. Head Neck (2004) 26(1):77-84. doi:10.1002/ hed. 10326

21. Etiz D, Orhan B, Demirüstü C, Ozdamar K, Cakmak A. Comparison of radiation-induced oral mucositis scoring systems. Tumori (2002) 88(5):379-84.

22. Riesenbeck D, Dorr W. Documentation of radiation-induced oral mucositis. Scoring systems. Strahlenther Onkol (1998) 174(Suppl 3):44-6.

23. Sonis ST, Eilers JP, Epstein JB, LeVeque FG, Liggett WH Jr, Mulagha MT, et al. Validation of a new scoring system for the assessment of clinical trial research of oral mucositis induced by radiation or chemotherapy. 
Mucositis Study Group. Cancer (1999) 85(10):2103-13. doi:10.1002/ (SICI) 1097-0142(19990515)85:10<2103::AID-CNCR2>3.0.CO;2-0

24. WCCNR. Assessing stomatitis: refinement of the Western Consortium for Cancer Nursing Research (WCCNR) stomatitis staging system. Can Oncol Nurs J (1998) 4:160-5.

25. Trotti A, Byhardt R, Stetz J, Gwede C, Corn B, Fu K, et al. Common toxicity criteria: version 2.0. An improved reference for grading the acute effects of cancer treatment: impact on radiotherapy. Int J Radiat Oncol Biol Phys (2000) 47:13-47. doi:10.1016/S0360-3016(99)00559-3

26. National Cancer Institute. Common Toxicity Criteria. Version 2.0. (1999). Available from: https://ctep.cancer.gov/protocoldevelopment/electronic_ applications/docs/ctcv20_4-30-992.pdf

27. McGuire DB, Peterson DE, Muller S, Owen DC, Slemmons MF, Schubert MM. The 20 item oral mucositis index: reliability and validity in bone marrow and stem cell transplant patients. Cancer Invest (2002) 20:893-903. doi:10.1081/ CNV-120005902

28. Sonis ST, Oster G, Fuchs F, Bellm L, Bradford WZ, Edelsberg J, et al. Oral mucositis and the clinical and economic outcomes of hematopoietic stem-cell transplantation. JClin Oncol (2001) 19:2201-5. doi:10.1200/ JCO.2001.19.8.2201

29. Parker L. Prevention and management of oral mucositis for an outpatient oncology setting. Okla Nurse (2005) 50:10-2.

30. Quinn B, Potting CM, Stone R, Blijlevens NM, Fliedner M, Margulies A, et al. Guidelines for the assessment of oral mucositis in adult chemotherapy, radiotherapy and haematopoietic stem cell transplant patients. Eur J Cancer (2008) 44(1):61-72. doi:10.1016/j.ejca.2007.09.014

31. Uçüncü H, Ertekin MV, Yörük O, Sezen O, Ozkan A, Erdoğan F, et al. Vitamin E and L-carnitine, separately or in combination, in the prevention of radiation-induced oral mucositis and myelosuppression: a controlled study in a rat model. J Radiat Res (2006) 47(1):91-102. doi:10.1269/ jrr.47.91

32. Schmidt W, Rainville LC, McEneff G, Sheehan D, Quinn B. A proteomic evaluation of the effects of the pharmaceuticals diclofenac and gemfibrozil on marine mussels (Mytilus spp.): evidence for chronic sublethal effects on stress-response proteins. Drug Test Anal (2014) 6(3):210-9. doi:10.1002/ dta. 1463

33. Lalla RV, Sonis ST, Peterson DE. Management of oral mucositis in patients who have cancer. Dent Clin North Am (2008) 52(1):61-77,viii. doi:10.1016/j. cden.2007.10.002

34. Lalla RV, Bowen J, Barasch A, Elting L, Epstein J, Keefe DM, et al. MASCC/ ISOO clinical practice guidelines for the management of mucositis secondary to cancer therapy. Cancer (2014) 120(10):1453-61. doi:10.1002/cncr.28592

35. Lalla RV. The MASCC/ISOO mucositis guidelines update: introduction to the first set of articles. Support Care Cancer (2013) 21(1):301-2. doi:10.1007/ s00520-012-1660-z

36. Lalla RV, Ashbury FD. The MASCC/ISOO mucositis guidelines: dissemination and clinical impact. Support Care Cancer (2013) 21(11):3161-3. doi:10.1007/s00520-013-1924-2

37. Watanabe S, Suemaru K, Nakanishi M, Nakajima N, Tanaka M, Tanaka A, et al. Assessment of the hamster cheek pouch as a model for radiationinduced oral mucositis, and evaluation of the protective effects of keratinocyte growth factor using this model. Int J Radiat Biol (2014) 90(10):884-91. doi:10.3109/09553002.2014.922716

38. Zheng C, Cotrim AP, Sunshine AN, Sugito T, Liu L, Sowers A, et al. Prevention of radiation-induced oral mucositis after adenoviral vector-mediated transfer of the keratinocyte growth factor cDNA to mouse submandibular glands. Clin Cancer Res (2009) 15(14):4641-8. doi:10.1158/1078-0432. CCR-09-0819

39. Kanuga S. Cryotherapy and keratinocyte growth factor may be beneficial in preventing oral mucositis in patients with cancer, and sucralfate is effective in reducing its severity. J Am Dent Assoc (2013) 144(8):928-9. doi:10.14219/ jada.archive.2013.0211

40. Sonis ST. Efficacy of palifermin (keratinocyte growth factor-1) in the amelioration of oral mucositis. Core Evid (2009) 4:199-205. doi:10.2147/CE.S5995

41. Tsirigotis P, Triantafyllou K, Girkas K, Giannopoulou V, Ioannidou E, Chondropoulos S, et al. Keratinocyte growth factor is effective in the prevention of intestinal mucositis in patients with hematological malignancies treated with high-dose chemotherapy and autologous hematopoietic
SCT: a video-capsule endoscopy study. Bone Marrow Transplant (2008) 42(5):337-43. doi:10.1038/bmt.2008.168

42. Blijlevens N, Sonis S. Palifermin (recombinant keratinocyte growth factor-1): a pleiotropic growth factor with multiple biological activities in preventing chemotherapy- and radiotherapy-induced mucositis. Ann Oncol (2007) 18(5):817-26. doi:10.1093/annonc/mdl332

43. Beaven AW, Shea TC. Recombinant human keratinocyte growth factor palifermin reduces oral mucositis and improves patient outcomes after stem cell transplant. Drugs Today (Barc) (2007) 43(7):461-73. doi:10.1358/ dot.2007.43.7.1119723

44. Borges L, Rex KL, Chen JN, Wei P, Kaufman S, Scully S, et al. A protective role for keratinocyte growth factor in a murine model of chemotherapy and radiotherapy-induced mucositis. Int J Radiat Oncol Biol Phys (2006) 66(1):254-62. doi:10.1016/j.ijrobp.2006.05.025

45. Beaven AW, Shea TC. Palifermin: a keratinocyte growth factor that reduces oral mucositis after stem cell transplant for haematological malignancies. Expert Opin Pharmacother (2006) 7(16):2287-99. doi:10.1517/14656566.7.16.2287

46. Dorr W, Reichel S, Spekl K. Effects of keratinocyte growth factor (palifermin) administration protocols on oral mucositis (mouse) induced by fractionated irradiation. Radiother Oncol (2005) 75(1):99-105. doi:10.1016/j. radonc.2004.12.006

47. Dörr W, Bässler S, Reichel S, Spekl K. Reduction of radiochemotherapyinduced early oral mucositis by recombinant human keratinocyte growth factor (palifermin): experimental studies in mice. Int J Radiat Oncol Biol Phys (2005) 62(3):881-7. doi:10.1016/j.ijrobp.2005.03.050

48. Lee D, Jain VK. The use of recombinant human keratinocyte growth factor (palifermin) to ameliorate treatment-induced mucositis. Support Cancer Ther (2003) 1(1):20-2. doi:10.1016/S1543-2912(13)60075-2

49. Potten CS, Booth D, Cragg NJ, Tudor GL, O'Shea JA, Booth C, et al. Cell kinetic studies in the murine ventral tongue epithelium: mucositis induced by radiation and its protection by pretreatment with keratinocyte growth factor (KGF). Cell Prolif (2002) 35(Suppl 1):32-47. doi:10.1046/j.1365-2184.35.s1.3.x

50. Gibson RJ, Keefe DM, Clarke JM, Regester GO, Thompson FM, Goland GJ, et al. The effect of keratinocyte growth factor on tumour growth and small intestinal mucositis after chemotherapy in the rat with breast cancer. Cancer Chemother Pharmacol (2002) 50(1):53-8. doi:10.1007/s00280002-0460-4

51. Farrell CL, Rex KL, Chen JN, Bready JV, DiPalma CR, Kaufman SA, et al. The effects of keratinocyte growth factor in preclinical models of mucositis. Cell Prolif (2002) 35(Suppl 1):78-85. doi:10.1046/j.1365-2184. 35.s1.8.x

52. Dorr W, Spekl K, Farrell CL. Amelioration of acute oral mucositis by keratinocyte growth factor: fractionated irradiation. Int J Radiat Oncol Biol Phys (2002) 54(1):245-51. doi:10.1016/S0360-3016(02)02918-8

53. Dörr W, Noack R, Spekl K, Farrell CL. Modification of oral mucositis by keratinocyte growth factor: single radiation exposure. Int J Radiat Biol (2001) 77(3):341-7. doi:10.1080/09553000010018873

54. Bardet E, Martin L, Calais G, Alfonsi M, Feham NE, Tuchais C, et al. Subcutaneous compared with intravenous administration of amifostine in patients with head and neck cancer receiving radiotherapy: final results of the GORTEC2000-02 phase III randomized trial. J Clin Oncol (2011) 29(2):127-33. doi:10.1200/JCO.2009.25.5638

55. Wasserman TH, Brizel DM, Henke M, Monnier A, Eschwege F, Sauer R, et al. Influence of intravenous amifostine on xerostomia, tumor control, and survival after radiotherapy for head-and-neck cancer: 2-year follow-up of a prospective, randomized, phase III trial. Int J Radiat Oncol Biol Phys (2005) 63(4):985-90. doi:10.1016/j.ijrobp.2005.07.966

56. Amrein PC, Clark JR, Supko JG, Fabian RL, Wang CC, Colevas AD, et al. Phase I trial and pharmacokinetics of escalating doses of paclitaxel and concurrent hyperfractionated radiotherapy with or without amifostine in patients with advanced head and neck carcinoma. Cancer (2005) 104(7):1418-27. doi:10.1002/cncr.21312

57. Komaki R, Lee JS, Milas L, Lee HK, Fossella FV, Herbst RS, et al. Effects of amifostine on acute toxicity from concurrent chemotherapy and radiotherapy for inoperable non-small-cell lung cancer: report of a randomized comparative trial. Int J Radiat Oncol Biol Phys (2004) 58(5):1369-77. doi:10.1016/j. ijrobp.2003.10.005 
58. Karacetin D, Yücel B, Leblebicioğlu B, Aksakal O, Maral O, Incekara O. A randomized trial of amifostine as radioprotector in the radiotherapy of head and neck cancer. J BUON (2004) 9(1):23-6.

59. Athanassiou H, Antonadou D, Coliarakis N, Kouveli A, Synodinou M, Paraskevaidis $\mathrm{M}$, et al. Protective effect of amifostine during fractionated radiotherapy in patients with pelvic carcinomas: results of a randomized trial. Int J Radiat Oncol Biol Phys (2003) 56(4):1154-60. doi:10.1016/ S0360-3016(03)00187-1

60. Bardet E, Martin L, Calais G, Tuchais C, Bourhis J, Rhein B, et al. Preliminary data of the GORTEC 2000-02 phase III trial comparing intravenous and subcutaneous administration of amifostine for head and neck tumors treated by external radiotherapy. Semin Oncol (2002) 29(6 Suppl 19):57-60. doi:10.1053/sonc.2002.37348

61. Li CJ, Wang SZ, Wang SY, Zhang YP. Assessment of the effect of local application of amifostine on acute radiation-induced oral mucositis in guinea pigs. J Radiat Res (2014) 55(5):847-54. doi:10.1093/jrr/rru024

62. Praetorius NP, Mandal TK. Alternate delivery route for amifostine as a radio-/chemo-protecting agent. J Pharm Pharmacol (2008) 60(7):809-15. doi:10.1211/jpp.60.7.0001

63. Kouvaris JR, Kouloulias VE, Vlahos LJ. Amifostine: the first selective-target and broad-spectrum radioprotector. Oncologist (2007) 12(6):738-47. doi:10.1634/theoncologist.12-6-738

64. Eisbruch A. Amifostine in the treatment of head and neck cancer: intravenous administration, subcutaneous administration, or none of the above. J Clin Oncol (2011) 29(2):119-21. doi:10.1200/JCO.2010.31.5051

65. Gu J, Zhu S, Li X, Wu H, Li Y, Hua F. Effect of amifostine in head and neck cancer patients treated with radiotherapy: a systematic review and metaanalysis based on randomized controlled trials. PLoS One (2014) 9(5):e95968. doi:10.1371/journal.pone.0095968

66. Keus R, Noach P, de Boer R, Lebesque J. The effect of customized beam shaping on normal tissue complications in radiation therapy of parotid gland tumors. Radiother Oncol (1991) 21(3):211-7. doi:10.1016/01678140(91)90039-J

67. Kaanders JH, Fleming TJ, Ang KK, Maor MH, Peters LJ. Devices valuable in head and neck radiotherapy. Int J Radiat Oncol Biol Phys (1992) 23(3):639-45. doi:10.1016/0360-3016(92)90023-B

68. Perch SJ, Machtay M, Markiewicz DA, Kligerman MM. Decreased acute toxicity by using midline mucosa-sparing blocks during radiation therapy for carcinoma of the oral cavity, oropharynx, and nasopharynx. Radiology (1995) 197(3):863-6. doi:10.1148/radiology.197.3.7480771

69. Kouloulias V, Thalassinou S, Platoni K, Zygogianni A, Kouvaris J, Antypas C, et al. The treatment outcome and radiation-induced toxicity for patients with head and neck carcinoma in the IMRT era: a systematic review with dosimetric and clinical parameters. Biomed Res Int (2013) 2013:401261. doi:10.1155/2013/401261

70. Bensadoun RJ, Franquin JC, Ciais G, Darcourt V, Schubert MM, Viot M, et al. Low-energy $\mathrm{He} / \mathrm{Ne}$ laser in the prevention of radiation-induced mucositis. A multicenter phase III randomized study in patients with head and neck cancer. Support Care Cancer (1999) 7(4):244-52. doi:10.1007/ s005200050256

71. Barber C, Powell R, Ellis A, Hewett J. Comparing pain control and ability to eat and drink with standard therapy vs Gelclair: a preliminary, double centre, randomised controlled trial on patients with radiotherapy-induced oral mucositis. Support Care Cancer (2007) 15(4):427-40. doi:10.1007/ s00520-006-0171-1

72. Klimberg VS, Souba WW, Dolson DJ, Salloum RM, Hautamaki RD, Plumley DA, et al. Prophylactic glutamine protects the intestinal mucosa from radiation injury. Cancer (1990) 66(1):62-8. doi:10.1002/10970142(19900701)66:1<62::AID-CNCR2820660113>3.0.CO;2-E

73. Huang EY, Leung SW, Wang CJ, Chen HC, Sun LM, Fang FM, et al. Oral glutamine to alleviate radiation-induced oral mucositis: a pilot randomized trial. Int J Radiat Oncol Biol Phys (2000) 46(3):535-9. doi:10.1016/ S0360-3016(99)00402-2

74. Peterson DE, Jones JB, Petit RG II. Randomized, placebo-controlled trial of Saforis for prevention and treatment of oral mucositis in breast cancer patients receiving anthracycline-based chemotherapy. Cancer (2007) 109(2):322-31. doi:10.1002/cncr.22384

75. Grumetto L, Del Prete A, Ortosecco G, Barbato F, Del Prete S, Borrelli A, et al. Study on the protective effect of a new manganese superoxide dismutase on the microvilli of rabbit eyes exposed to UV radiation. Biomed Res Int (2015) 2015:973197. doi:10.1155/2015/973197

76. Eldridge A, Fan M, Woloschak G, Grdina DJ, Chromy BA, Li JJ. Manganese superoxide dismutase interacts with a large scale of cellular and mitochondrial proteins in low-dose radiation-induced adaptive radioprotection. Free Radic Biol Med (2012) 53(10):1838-47. doi:10.1016/j. freeradbiomed.2012.08.589

77. Rajagopalan MS, Stone B, Rwigema JC, Salimi U, Epperly MW, Goff J, et al. Intraesophageal manganese superoxide dismutase-plasmid liposomes ameliorates novel total-body and thoracic radiation sensitivity of NOS1-/- mice. Radiat Res (2010) 174(3):297-312. doi:10.1667/RR2019.1

78. Holley AK, Xu Y, St Clair DK, St Clair WH. RelB regulates manganese superoxide dismutase gene and resistance to ionizing radiation of prostate cancer cells. Ann N Y Acad Sci (2010) 1201:129-36. doi:10.1111/j.1749-6632.2010.05613.x

79. dos Santos Montagner GF, Sagrillo M, Machado MM, Almeida RC, Mostardeiro CP, Duarte MM, et al. Toxicological effects of ultraviolet radiation on lymphocyte cells with different manganese superoxide dismutase Ala16Val polymorphism genotypes. Toxicol In Vitro (2010) 24(5):1410-6. doi:10.1016/j.tiv.2010.04.010

80. Holley AK, St Clair DK. Preventing Dr. Jekyll from becoming Mr. Hyde: is manganese superoxide dismutase the key to prevent radiation-induced neoplastic transformation? Cancer Biol Ther (2009) 8(20):1972-3. doi:10.4161/ cbt.8.20.9941

81. Josson S, Xu Y, Fang F, Dhar SK, St Clair DK, St Clair WH. RelB regulates manganese superoxide dismutase gene and resistance to ionizing radiation of prostate cancer cells. Oncogene (2006) 25(10):1554-9. doi:10.1038/ sj.onc. 1209186

82. Guo HL, Zhao HW, Xu ZF, Ma H, Song XL, Guan J, et al. [Manganese superoxide dismutase gene transfection of mouse small intestinal epithelial cells protects them from radiation injury]. Zhonghua Zhong Liu Za Zhi (2005) 27(11):672-5.

83. Guo HL, Wolfe D, Epperly MW, Huang S, Liu K, Glorioso JC, et al. Gene transfer of human manganese superoxide dismutase protects small intestinal villi from radiation injury. J Gastrointest Surg (2003) 7(2):229-35; discussion 235-6.

84. Guo H, Seixas-Silva JA Jr, Epperly MW, Gretton JE, Shin DM, Bar-Sagi D, et al. Prevention of radiation-induced oral cavity mucositis by plasmid/ liposome delivery of the human manganese superoxide dismutase (SOD2) transgene. Radiat Res (2003) 159(3):361-70. doi:10.1667/0033-7587(2003)1 59[0361:PORIOC]2.0.CO;2

85. Guo G, Yan-Sanders Y, Lyn-Cook BD, Wang T, Tamae D, Ogi J, et al. Manganese superoxide dismutase-mediated gene expression in radiationinduced adaptive responses. Mol Cell Biol (2003) 23(7):2362-78. doi:10.1128/ MCB.23.7.2362-2378.2003

86. Epperly MW, Bernarding M, Gretton J, Jefferson M, Nie S, Greenberger JS. Overexpression of the transgene for manganese superoxide dismutase (MnSOD) in 32D cl 3 cells prevents apoptosis induction by TNF-alpha, IL-3 withdrawal, and ionizing radiation. Exp Hematol (2003) 31(6):465-74. doi:10.1016/S0301-472X(03)00041-9

87. Epperly MW, Sikora CA, DeFilippi SJ, Gretton JA, Zhan Q, Kufe DW, et al. Manganese superoxide dismutase (SOD2) inhibits radiation-induced apoptosis by stabilization of the mitochondrial membrane. Radiat Res (2002) 157(5):568-77. doi:10.1667/0033-7587(2002)157[0568: MSDSIR]2.0.CO;2

88. Motoori S, Majima HJ, Ebara M, Kato H, Hirai F, Kakinuma S, et al. Overexpression of mitochondrial manganese superoxide dismutase protects against radiation-induced cell death in the human hepatocellular carcinoma cell line HLE. Cancer Res (2001) 61(14):5382-8.

89. Epperly MW, Kagan VE, Sikora CA, Gretton JE, Defilippi SJ, Bar-Sagi D, et al. Manganese superoxide dismutase-plasmid/liposome (MnSOD-PL) administration protects mice from esophagitis associated with fractionated radiation. Int J Cancer (2001) 96(4):221-31. doi:10.1002/ijc.1023

90. Epperly MW, Gretton JA, DeFilippi SJ, Greenberger JS, Sikora CA, Liggitt D, et al. Modulation of radiation-induced cytokine elevation associated with esophagitis and esophageal stricture by manganese superoxide dismutase-plasmid/liposome (SOD2-PL) gene therapy. Radiat Res (2001) 155(1 Pt 1):2-14. doi:10.1667/0033-7587(2001)155[0002:MORICE]2.0.CO;2

91. Kuninaka S, Ichinose Y, Koja K, Toh Y. Suppression of manganese superoxide dismutase augments sensitivity to radiation, hyperthermia and 
doxorubicin in colon cancer cell lines by inducing apoptosis. Br J Cancer (2000) 83(7):928-34. doi:10.1054/bjoc.2000.1367

92. Epperly MW, Epstein CJ, Travis EL, Greenberger JS. Decreased pulmonary radiation resistance of manganese superoxide dismutase (MnSOD)-deficient mice is corrected by human manganese superoxide dismutase-plasmid/ liposome (SOD2-PL) intratracheal gene therapy. Radiat Res (2000) 154(4):365-74. doi:10.1667/0033-7587(2000)154[0365:DPRROM]2.0.CO;2

93. Sasaki H, Akamatsu H, Horio T. Effects of a single exposure to UVB radiation on the activities and protein levels of copper-zinc and manganese superoxide dismutase in cultured human keratinocytes. Photochem Photobiol (1997) 65(4):707-13. doi:10.1111/j.1751-1097.1997.tb01914.x

94. Otero G, Avila MA, Emfietzoglou D, Clerch LB, Massaro D, Notario V. Increased manganese superoxide dismutase activity, protein, and mRNA levels and concurrent induction of tumor necrosis factor alpha in radiationinitiated Syrian hamster cells. Mol Carcinog (1996) 17(4):175-80. doi:10.1002/ (SICI) 1098-2744(199612)17:4<175::AID-MC1>3.0.CO;2-D

95. Nakano T, Oka K, Taniguchi N. Manganese superoxide dismutase expression correlates with $\mathrm{p} 53$ status and local recurrence of cervical carcinoma treated with radiation therapy. Cancer Res (1996) 56(12):2771-5.

96. Urano M, Kuroda M, Reynolds R, Oberley TD, St Clair DK. Expression of manganese superoxide dismutase reduces tumor control radiation dose: gene-radiotherapy. Cancer Res (1995) 55(12):2490-3.

97. Lin PS, Ho KC, Sung SJ, Tsai S. Cytotoxicity and manganese superoxide dismutase induction by tumor necrosis factor-alpha and ionizing radiation in MCF-7 human breast carcinoma cells. Lymphokine Cytokine Res (1993) 12(5):303-8.

98. Hirose K, Longo DL, Oppenheim JJ, Matsushima K. Overexpression of mitochondrial manganese superoxide dismutase promotes the survival of tumor cells exposed to interleukin-1, tumor necrosis factor, selected anticancer drugs, and ionizing radiation. FASEB J (1993) 7(2):361-8.

99. LeVequeFG, Parzuchowski JB, Farinacci GC, Redding SW, Rodu B, Johnson JT, et al. Clinical evaluation of MGI 209, an anesthetic, film-forming agent for relief from painful oral ulcers associated with chemotherapy. J Clin Oncol (1992) 10(12):1963-8. doi:10.1200/JCO.1992.10.12.1963

100. Barker G, Loftus L, Cuddy P, Barker B. The effects of sucralfate suspension and diphenhydramine syrup plus kaolin-pectin on radiotherapyinduced mucositis. Oral Surg Oral Med Oral Pathol (1991) 71(3):288-93. doi:10.1016/0030-4220(91)90301-R

101. Su YX, Benedek GA, Sieg P, Liao GQ, Dendorfer A, Meller B, et al. Radioprotective effect of lidocaine on neurotransmitter agonist-induced secretion in irradiated salivary glands. PLoS One (2013) 8(3):e60256. doi:10.1371/journal.pone.0060256

102. Carnel SB, Blakeslee DB, Oswald SG, Barnes M. Treatment of radiation- and chemotherapy-induced stomatitis. Otolaryngol Head Neck Surg (1990) 102(4):326-30. doi:10.1177/019459989010200404

103. Rodu B, Russell CM, Ray KL. Treatment of oral ulcers with hydroxypropylcellulose film (Zilactin). Compendium (1988) 9(5):420-2.

104. Sung L, Robinson P, Treister N, Baggott T, Gibson P, Tissing W, et al. Guideline for the prevention of oral and oropharyngeal mucositis in children receiving treatment for cancer or undergoing haematopoietic stem cell transplantation. BMJ Support Palliat Care (2017) 7(1):7-16. doi:10.1136/ bmjspcare-2014-000804

105. Miller MM, Donald DV, Hagemann TM. Prevention and treatment of oral mucositis in children with cancer. J Pediatr Pharmacol Ther (2012) 17(4):340-50. doi:10.5863/1551-6776-17.4.340

106. Ps SK, Balan A, Sankar A, Bose T. Radiation induced oral mucositis. Indian J Palliat Care (2009) 15(2):95-102. doi:10.4103/0973-1075.58452

107. Sarvizadeh M, Hemati S, Meidani M, Ashouri M, Roayaei M, Shahsanai A. Morphine mouthwash for the management of oral mucositis in patients with head and neck cancer. Adv Biomed Res (2015) 4:44. doi:10.4103/2277-9175.151254

108. Vayne-Bossert P, Escher M, de Vautibault CG, Dulguerov P, Allal A, Desmeules J, et al. Effect of topical morphine (mouthwash) on oral pain due to chemotherapy- and/or radiotherapy-induced mucositis: a randomized double-blinded study. J Palliat Med (2010) 13(2):125-8. doi:10.1089/ jpm.2009.0195

109. Rothwell BR, Spektor WS. Palliation of radiation-related mucositis. Spec Care Dentist (1990) 10(1):21-5. doi:10.1111/j.1754-4505.1990.tb01082.x
110. Murata Y, Kofuji K, Nishida N, Kamaguchi R. Development of film dosage form containing allopurinol for prevention and treatment of oral mucositis. ISRN Pharm (2012) 2012:764510. doi:10.5402/2012/764510

111. Tomoda K, Asahiyama M, Ohtsuki E, Nakajima T, Terada H, Kanebako $\mathrm{M}$, et al. Preparation and properties of carrageenan microspheres containing allopurinol and local anesthetic agents for the treatment of oral mucositis. Colloids Surf B Biointerfaces (2009) 71(1):27-35. doi:10.1016/j. colsurfb.2009.01.003

112. Kitagawa J, Nasu M, Okumura H, Shibata A, Makino K, Terada H, et al. Allopurinol gel mitigates radiation-induced mucositis and dermatitis. J Radiat Res (2008) 49(1):49-54. doi:10.1269/jrr.07038

113. Loprinzi CL, Burnham N. Allopurinol mouthwash as prophylactic therapy for 5-fluorouracil-induced mucositis. Eur J Surg Oncol (1989) 15(3):297.

114. Renck D, Santos AA Jr, Machado P, Petersen GO, Lopes TG, Santos DS, et al. Human uridine phosphorylase-1 inhibitors: a new approach to ameliorate 5-fluorouracil-induced intestinal mucositis. Invest New Drugs (2014) 32(6):1301-7. doi:10.1007/s10637-014-0135-0

115. PanahiY,AlaS,SaeediM,OkhovatianA,BazzazN,NaghizadehMM.Allopurinol mouth rinse for prophylaxis of fluorouracil-induced mucositis. Eur J Cancer Care (Engl) (2010) 19(3):308-12. doi:10.1111/j.1365-2354.2008.01042.x

116. Seiter K, Kemeny N, Martin D, Schneider A, Williams L, Colofiore J, et al. Uridine allows dose escalation of 5-fluorouracil when given with $\mathrm{N}$-phosphonacetyl-L-aspartate, methotrexate, and leucovorin. Cancer (1993) 71(5):1875-81. doi:10.1002/1097-0142(19930301)71:5<1875::AID-CNCR2820710526>3.0.CO;2-9

117. Foote RL, Loprinzi CL, Frank AR, O’Fallon JR, Gulavita S, Tewfik HH, et al. Randomized trial of a chlorhexidine mouthwash for alleviation of radiation-induced mucositis. J Clin Oncol (1994) 12(12):2630-3. doi:10.1200/ JCO.1994.12.12.2630

118. Roopashri G, Jayanthi K, Guruprasad R. Efficacy of benzydamine hydrochloride, chlorhexidine, and povidone iodine in the treatment of oral mucositis among patients undergoing radiotherapy in head and neck malignancies: a drug trail. Contemp Clin Dent (2011) 2(1):8-12. doi:10.4103/0976-237X.79292

119. Dodd MJ, Larson PJ, Dibble SL, Miaskowski C, Greenspan D, MacPhail L, et al. Randomized clinical trial of chlorhexidine versus placebo for prevention of oral mucositis in patients receiving chemotherapy. Oncol Nurs Forum (1996) 23(6):921-7.

120. de Boer-Dennert MM, Batchelor D. ["Randomized clinical trial of chlorhexidine versus placebo for prevention of oral mucositis in patients receiving chemotherapy". Marylin J. Dodd et al. Report of discussion of this article in the IKA Nursing Research Utilization Board]. Oncologica (1997) 14(3):16-8.

121. Davies AN, Singer J. A comparison of artificial saliva and pilocarpine in radiation-induced xerostomia. J Laryngol Otol (1994) 108(8):663-5. doi:10.1017/S0022215100127768

122. Dos Reis PE, Ciol MA, de Melo NS, Figueiredo PT, Leite AF, Manzi Nde M. Chamomile infusion cryotherapy to prevent oral mucositis induced by chemotherapy: a pilot study. Support Care Cancer (2016) 24(10):4393-8. doi:10.1007/s00520-016-3279-y

123. Curra M, Martins MA, Lauxen IS, Pellicioli AC, Sant'Ana Filho M, Pavesi VC, et al. Effect of topical chamomile on immunohistochemical levels of IL-1beta and TNF-alpha in 5-fluorouracil-induced oral mucositis in hamsters. Cancer Chemother Pharmacol (2013) 71(2):293-9. doi:10.1007/s00280-012-2013-9

124. Pavesi VC, Lopez TC, Martins MA, Sant'Ana Filho M, Bussadori SK, Fernandes KP, et al. Healing action of topical chamomile on 5-fluoracil induced oral mucositis in hamster. Support Care Cancer (2011) 19(5):639-46. doi:10.1007/s00520-010-0875-0

125. Mazokopakis EE, Vrentzos GE, Papadakis JA, Babalis DE, Ganotakis ES, Wild chamomile (Matricaria recutita L.) mouthwashes in methotrexateinduced oral mucositis. Phytomedicine (2005) 12(1-2):25-7. doi:10.1016/j. phymed.2003.11.003

126. Fidler P, Loprinzi CL, O’Fallon JR, Leitch JM, Lee JK, Hayes DL, et al. Prospective evaluation of a chamomile mouthwash for prevention of 5-FU-induced oral mucositis. Cancer (1996) 77(3):522-5. doi:10.1002/ (SICI) 1097-0142(19960201)77:3<522::AID-CNCR14>3.0.CO;2-6

127. Van den Wyngaert $T$. Topical honey application to reduce radiation-induced oral mucositis: a therapy too sweet to ignore? J Evid Based Dent Pract (2012) 12(4):203-5. doi:10.1016/j.jebdp.2012.09.011 
128. Song JJ, Twumasi-Ankrah P, Salcido R. Systematic review and meta-analysis on the use of honey to protect from the effects of radiation-induced oral mucositis. Adv Skin Wound Care (2012) 25(1):23-8. doi:10.1097/01. ASW.0000410687.14363.a3

129. Khanal B, Baliga M, Uppal N. Effect of topical honey on limitation of radiation-induced oral mucositis: an intervention study. Int J Oral Maxillofac Surg (2010) 39(12):1181-5. doi:10.1016/j.ijom.2010.05.014

130. Bardy J, Molassiotis A, Ryder WD, Mais K, Sykes A, Yap B, et al. A double-blind, placebo-controlled, randomised trial of active manuka honey and standard oral care for radiation-induced oral mucositis. $\mathrm{Br} \mathrm{JOral}$ Maxillofac Surg (2012) 50(3):221-6. doi:10.1016/j.bjoms.2011.03.005

131. Santos-Silva AR, Rosa GB, Eduardo CP, Dias RB, Brandao TB. Increased risk for radiation-related caries in cancer patients using topical honey for the prevention of oral mucositis. Int J Oral Maxillofac Surg (2011) 40(11):1335-6; author reply 1235. doi:10.1016/j.ijom.2011.05.006

132. Arora H, Pai KM, Maiya A, Vidyasagar MS, Rajeev A. Efficacy of He-Ne laser in the prevention and treatment of radiotherapy-induced oral mucositis in oral cancer patients. Oral Surg Oral Med Oral Pathol Oral Radiol Endod (2008) 105(2):180-6, 186.e1. doi:10.1016/j.tripleo.2007.07.043

133. Hawley P, Hovan A, McGahan CE, Saunders D. A randomized placebocontrolled trial of manuka honey for radiation-induced oral mucositis. Support Care Cancer (2014) 22(3):751-61. doi:10.1007/s00520-013-2031-0

134. Ala S, Saeedi M, Janbabai G, Ganji R, Azhdari E, Shiva A. Efficacy of sucralfate mouth wash in prevention of 5-fluorouracil induced oral mucositis: a prospective, randomized, double-blind, controlled trial. Nutr Cancer (2016) 68(3):456-63. doi:10.1080/01635581.2016.1153666

135. Nottage M, McLachlan SA, Brittain MA, Oza A, Hedley D, Feld R, et al. Sucralfate mouthwash for prevention and treatment of 5-fluorouracilinduced mucositis: a randomized, placebo-controlled trial. Support Care Cancer (2003) 11(1):41-7. doi:10.1007/s00520-002-0378-8

136. Dodd MJ, Miaskowski C, Greenspan D, MacPhail L, Shih AS, Shiba G, et al. Radiation-induced mucositis: a randomized clinical trial of micronized sucralfate versus salt \& soda mouthwashes. Cancer Invest (2003) 21(1):21-33. doi:10.1081/CNV-120016400

137. Saarilahti K, Kajanti M, Joensuu T, Kouri M, Joensuu H. Comparison of granulocyte-macrophage colony-stimulating factor and sucralfate mouthwashes in the prevention of radiation-induced mucositis: a double-blind prospective randomized phase III study. Int J Radiat Oncol Biol Phys (2002) 54(2):479-85. doi:10.1016/S0360-3016(02)02935-8

138. Kilic D, Akcali Z. Comment on: granulocyte macrophage-colony stimulating factor (GM-CSF) and sucralfate in prevention of radiation-induced mucositis: a prospective randomized study. Int J Radiat Oncol Biol Phys (2001) 50(5):1373-4. doi:10.1016/S0360-3016(01)01587-5

139. Castagna L, Benhamou E, Pedraza E, Luboinski M, Forni M, Brandes I, et al. Prevention of mucositis in bone marrow transplantation: a double blind randomised controlled trial of sucralfate. Ann Oncol (2001) 12(7):953-5. doi:10.1023/A:1011119721267

140. Makkonen TA, Minn H, Jekunen A, Vilja P, Tuominen J, Joensuu H. Granulocyte macrophage-colony stimulating factor (GM-CSF) and sucralfate in prevention of radiation-induced mucositis: a prospective randomized study. Int J Radiat Oncol Biol Phys (2000) 46(3):525-34. doi:10.1016/ S0360-3016(99)00452-6

141. Etiz D, Erkal HS, Serin M, Küçük B, Hepari A, Elhan AH, et al. Clinical and histopathological evaluation of sucralfate in prevention of oral mucositis induced by radiation therapy in patients with head and neck malignancies. Oral Oncol (2000) 36(1):116-20. doi:10.1016/S1368-8375 (99)00075-5

142. Cengiz M, Ozyar E, Oztürk D, Akyol F, Atahan IL, Hayran M. Sucralfate in the prevention of radiation-induced oral mucositis. J Clin Gastroenterol (1999) 28(1):40-3. doi:10.1097/00004836-199901000-00009

143. Sur RK. Sucralfate in radiation-induced mucositis. S Afr Med J (1997) 87(3):337-8.

144. Meredith R, Salter M, Kim R, Spencer S, Weppelmann B, Rodu B, et al. Sucralfate for radiation mucositis: results of a double-blind randomized trial. Int JRadiat Oncol Biol Phys (1997) 37(2):275-9. doi:10.1016/ S0360-3016(96)00531-7

145. Franzén L, Henriksson R, Littbrand B, Zackrisson B. Effects of sucralfate on mucositis during and following radiotherapy of malignancies in the head and neck region. A double-blind placebo-controlled study. Acta Oncol (1995) 34(2):219-23. doi:10.3109/02841869509093959

146. Allison RR, Vongtama V, Vaughan J, Shin KH. Symptomatic acute mucositis can be minimized or prophylaxed by the combination of sucralfate and fluconazole. Cancer Invest (1995) 13(1):16-22. doi:10.3109/07357909509024890

147. Makkonen TA, Boström P, Vilja P, Joensuu H. Sucralfate mouth washing in the prevention of radiation-induced mucositis: a placebo-controlled double-blind randomized study. Int JRadiat Oncol Biol Phys (1994) 30(1):177-82. doi:10.1016/0360-3016(94)90533-9

148. Epstein JB, Wong FL. The efficacy of sucralfate suspension in the prevention of oral mucositis due to radiation therapy. Int J Radiat Oncol Biol Phys (1994) 28(3):693-8. doi:10.1016/0360-3016(94)90195-3

149. Shenep JL, Kalwinsky DK, Hutson PR, George SL, Dodge RK, Blankenship KR, et al. Efficacy of oral sucralfate suspension in prevention and treatment of chemotherapy-induced mucositis. JPediatr (1988) 113(4):758-63. doi:10.1016/S0022-3476(88)80397-4

150. Theodore C, Thurninger O, Hermitte H. [Radiation-induced mucositis: a new indication of sucralfate?]. Gastroenterol Clin Biol (1987) 11(4):345.

151. Solomon MA. Oral sucralfate suspension for mucositis. N Engl J Med (1986) 315(7):459-60. doi:10.1056/NEJM198608143150717

152. High KP, Legault C, Sinclair JA, Cruz J, Hill K, Hurd DD. Low plasma concentrations of retinol and alpha-tocopherol in hematopoietic stem cell transplant recipients: the effect of mucositis and the risk of infection. Am J Clin Nutr (2002) 76(6):1358-66.

153. Cohen G, Elad S, Or R, Galili D, Garfunkel AA. The use of tretinoin as oral mucositis prophylaxis in bone marrow transplantation patients: a preliminary study. Oral Dis (1997) 3(4):243-6. doi:10.1111/j.1601-0825.1997.tb00049.x

154. Ferreira PR, Fleck JF, Diehl A, Barletta D, Braga-Filho A, Barletta A, et al. Protective effect of alpha-tocopherol in head and neck cancer radiationinduced mucositis: a double-blind randomized trial. Head Neck (2004) 26(4):313-21. doi:10.1002/hed.10382

155. Oshitani T, Okada K, Kushima T, Suematsu T, Obayashi K, Hirata Y, et al. [Clinical evaluation of sodium alginate on oral mucositis associated with radiotherapy]. Nihon Gan Chiryo Gakkai Shi (1990) 25(6):1129-37.

156. Epstein JB, Stevenson-Moore P, Jackson S, Mohamed JH, Spinelli JJ. Prevention of oral mucositis in radiation therapy: a controlled study with benzydamine hydrochloride rinse. Int J Radiat Oncol Biol Phys (1989) 16(6):1571-5. doi:10.1016/0360-3016(89)90964-4

157. Epstein JB, Stevenson-Moore P. Benzydamine hydrochloride in prevention and management of pain in oral mucositis associated with radiation therapy. Oral Surg Oral Med Oral Pathol (1986) 62(2):145-8. doi:10.1016/0030-4220(86)90035-6

158. Rahn R, Adamietz IA, Boettcher HD, Schaefer V, Reimer K, Fleischer W. Povidone-iodine to prevent mucositis in patients during antineoplastic radiochemotherapy. Dermatology (1997) 195(Suppl 2):57-61. doi:10.1159/000246032

159. Adamietz IA, Rahn R, Böttcher HD, Schäfer V, Reimer K, Fleischer W. Prophylaxis with povidone-iodine against induction of oral mucositis by radiochemotherapy. Support Care Cancer (1998) 6(4):373-7. doi:10.1007/ s005200050179

160. Vokurka S, Bystricka E, Koza V, Scudlova J, Pavlicova V, Valentova D, et al. The comparative effects of povidone-iodine and normal saline mouthwashes on oral mucositis in patients after high-dose chemotherapy and APBSCT results of a randomized multicentre study. Support Care Cancer (2005) 13(7):554-8. doi:10.1007/s00520-005-0792-9

161. Berger A, Henderson M, Nadoolman W, Duffy V, Cooper D, Saberski L, et al. Oral capsaicin provides temporary relief for oral mucositis pain secondary to chemotherapy/radiation therapy. J Pain Symptom Manage (1995) 10(3):243-8. doi:10.1016/0885-3924(94)00130-D

162. Lalla RV, Pilbeam CC, Walsh SJ, Sonis ST, Keefe DM, Peterson DE. Role of the cyclooxygenase pathway in chemotherapy-induced oral mucositis: a pilot study. Support Care Cancer (2010) 18(1):95-103. doi:10.1007/ s00520-009-0635-1

163. Lopes NN, Plapler H, Chavantes MC, Lalla RV, Yoshimura EM, Alves MT. Cyclooxygenase-2 and vascular endothelial growth factor expression in 5-fluorouracil-induced oral mucositis in hamsters: evaluation of two low-intensity laser protocols. Support Care Cancer (2009) 17(11):1409-15. doi:10.1007/s00520-009-0603-9 
164. Sonis ST, O'Donnell KE, Popat R, Bragdon C, Phelan S, Cocks D, et al. The relationship between mucosal cyclooxygenase-2 (COX-2) expression and experimental radiation-induced mucositis. Oral Oncol (2004) 40(2):170-6. doi:10.1016/S1368-8375(03)00148-9

165. Pillsbury HC III, Webster WP, Rosenman J. Prostaglandin inhibitor and radiotherapy in advanced head and neck cancers. Arch Otolaryngol Head Neck Surg (1986) 112(5):552-3. doi:10.1001/archotol.1986.03780050076013

166. Kono T, Kaneko A, Matsumoto C, Miyagi C, Ohbuchi K, Mizuhara Y, et al. Multitargeted effects of hangeshashinto for treatment of chemotherapyinduced oral mucositis on inducible prostaglandin E2 production in human oral keratinocytes. Integr Cancer Ther (2014) 13(5):435-45. doi: $10.1177 / 1534735413520035$

167. Hanson WR, Marks JE, Reddy SP, Simon S, Mihalo WE, Tova Y. Protection from radiation-induced oral mucositis by a mouth rinse containing the prostaglandin E1 analog, misoprostol: a placebo controlled double blind clinical trial. Adv Exp Med Biol (1997) 400B:811-8.

168. Maltoni M, Sansoni E, Derni S, Milandri C, Martini F, Nanni O, et al. Topical prostaglandin E2 and chemo- and radio-induced oral mucositis. Oncol Rep (1996) 3(1):205-8

169. Hanson WR, Marks JE, Reddy SP, Simon S, Mihalo WE, Tova Y. Protection from radiation-induced oral mucositis by misoprostol, a prostaglandin $\mathrm{E}(1)$ analog: a placebo-controlled, double-blind clinical trial. Am J Ther (1995) 2(11):850-7. doi:10.1097/00045391-199511000-00005

170. Labar B, Mrsić M, Pavletić Z, Bogdanić V, Nemet D, Aurer I, et al. Prostaglandin E2 for prophylaxis of oral mucositis following BMT. Bone Marrow Transplant (1993) 11(5):379-82.

171. Matejka M, Nell A, Kment G, Schein A, Leukauf M, Porteder H, et al. Local benefit of prostaglandin E2 in radiochemotherapy-induced oral mucositis. Br J Oral Maxillofac Surg (1990) 28(2):89-91. doi:10.1016/0266-4356(90) 90128-8

172. Pretnar J, Glazar D, Mlakar U, Modic M. Prostaglandin E2 in the treatment of oral mucositis due to radiochemotherapy in patients with haematological malignancies. Bone Marrow Transplant (1989) 4(Suppl 3):106.

173. He D, Behar S, Roberts JE, Lim HW. The effect of L-cysteine and $\mathrm{N}$-acetylcysteine on porphyrin/heme biosynthetic pathway in cells treated with 5-aminolevulinic acid and exposed to radiation. Photodermatol Photoimmunol Photomed (1996) 12(5):194-9. doi:10.1111/j.1600-0781.1996. tb00199.x

174. Baier JE, Neumann HA, Moeller T, Kissler M, Borchardt D, Ricken D. [Radiation protection through cytokine release by $\mathrm{N}$-acetylcysteine]. Strahlenther Onkol (1996) 172(2):91-8.

175. Ozgur E, Sahin D, Tomruk A, Guler G, Sepici Dinçel A, Altan N, et al. The effects of $\mathrm{N}$-acetylcysteine and epigallocatechin-3-gallate on liver tissue protein oxidation and antioxidant enzyme levels after the exposure to radiofrequency radiation. Int J Radiat Biol (2015) 91(2):187-93. doi:10.3109/0955 3002.2015.966210

176. Li J, Meng Z, Zhang G, Xing Y, Feng L, Fan S, et al. $N$-acetylcysteine relieves oxidative stress and protects hippocampus of rat from radiation-induced apoptosis by inhibiting caspase-3. Biomed Pharmacother (2015) 70:1-6. doi:10.1016/j.biopha.2014.12.029

177. Kilciksiz S, Demirel C, Evirgen Ayhan S, Erdal N, Gurgul S, Tamer L, et al. $\mathrm{N}$-acetylcysteine ameliorates nitrosative stress on radiation-inducible damage in rat liver. J BUON (2011) 16(1):154-9.

178. Demirel C, Kilciksiz S, Gurgul S, Erdal N, Yildiz A. N-acetylcysteine ameliorates gamma-radiation-induced deterioration of bone quality in the rat femur. J Int Med Res (2011) 39(6):2393-401. doi:10.1177/147323001103900640

179. Wang Y, Zhang ZZ, Chen SQ, Zou ZD, Tu XH, Wang L. [Protective effect of $\mathrm{N}$-acetylcysteine on the intestinal barrier dysfunction after radiation injury in rats]. Zhonghua Wei Chang Wai Ke Za Zhi (2010) 13(3):219-22.

180. Demirel C, Kilciksiz S, Evirgen-Ayhan S, Gurgul S, Erdal N. The preventive effect of $N$-acetylcysteine on radiation-induced dermatitis in a rat model. J BUON (2010) 15(3):577-82.

181. Demirel C, Kilçiksiz S, Ay OI, Gürgül S, Ay ME, Erdal N. Effect of $\mathrm{N}$-acetylcysteine on radiation-induced genotoxicity and cytotoxicity in rat bone marrow. J Radiat Res (2009) 50(1):43-50. doi:10.1269/jrr.08066

182. Wu W, Abraham L, Ogony J, Matthews R, Goldstein G, Ercal N. Effects of $\mathrm{N}$-acetylcysteine amide (NACA), a thiol antioxidant on radiation-induced cytotoxicity in Chinese hamster ovary cells. Life Sci (2008) 82(21-22):112230. doi:10.1016/j.lfs.2008.03.016
183. Mansour HH, Hafez HF, Fahmy NM, Hanafi N. Protective effect of $\mathrm{N}$-acetylcysteine against radiation induced DNA damage and hepatic toxicity in rats. Biochem Pharmacol (2008) 75(3):773-80. doi:10.1016/j.bcp. 2007.09.018

184. Low WK, Sun L, Tan MG, Chua AW, Wang DY. L-N-acetylcysteine protects against radiation-induced apoptosis in a cochlear cell line. Acta Otolaryngol (2008) 128(4):440-5. doi:10.1080/00016480701762490

185. Kilciksiz S, Demirel C, Erdal N, Gürgül S, Tamer L, Ayaz L, et al. The effect of $\mathrm{N}$-acetylcysteine on biomarkers for radiation-induced oxidative damage in a rat model. Acta Med Okayama (2008) 62(6):403-9.

186. Sminia P, van der Kracht AH, Frederiks WM, Jansen W. Hyperthermia, radiation carcinogenesis and the protective potential of vitamin $\mathrm{A}$ and $N$-acetylcysteine. J Cancer Res Clin Oncol (1996) 122(6):343-50. doi:10.1007/ BF01220801

187. Tarbell NJ, Rosenblatt M, Amato DA, Hellman S. The effect of $N$-acetylcysteine inhalation on the tolerance to thoracic radiation in mice. Radiother Oncol (1986) 7(1):77-80. doi:10.1016/S0167-8140(86)80126-8

188. Kim JA, Baker DG, Hahn SS, Goodchild NT, Constable WC. Topical use of $\mathrm{N}$-acetylcysteine for reduction of skin reaction to radiation therapy. Semin Oncol (1983) 10(1 Suppl 1):86-92.

189. Lieschke GJ, Ramenghi U, O’Connor MP, Sheridan W, Szer J, Morstyn G. Studies of oral neutrophil levels in patients receiving G-CSF after autologous marrow transplantation. Br J Haematol (1992) 82(3):589-95. doi:10.111 1/j.1365-2141.1992.tb06472.x

190. Dazzi C, Cariello A, Giovanis P, Monti M, Vertogen B, Leoni M, et al. Prophylaxis with GM-CSF mouthwashes does not reduce frequency and duration of severe oral mucositis in patients with solid tumors undergoing high-dose chemotherapy with autologous peripheral blood stem cell transplantation rescue: a double blind, randomized, placebo-controlled study. Ann Oncol (2003) 14(4):559-63.

191. Cartee L, Petros WP, Rosner GL, Gilbert C, Moore S, Affronti ML, et al. Evaluation of GM-CSF mouthwash for prevention of chemotherapy-induced mucositis: a randomized, double-blind, dose-ranging study. Cytokine (1995) 7(5):471-7. doi:10.1006/cyto.1995.0064

192. McAleese JJ, Bishop KM, A’Hern R, Henk JM. Randomized phase II study of GM-CSF to reduce mucositis caused by accelerated radiotherapy of laryngeal cancer. Br J Radiol (2006) 79(943):608-13. doi:10.1259/bjr/55190439

193. Kannan V, Bapsy PP, Anantha N, Doval DC, Vaithianathan H, Banumathy G, et al. Efficacy and safety of granulocyte macrophage-colony stimulating factor (GM-CSF) on the frequency and severity of radiation mucositis in patients with head and neck carcinoma. Int J Radiat Oncol Biol Phys (1997) 37(5):1005-10. doi:10.1016/S0360-3016(97)00105-3

194. Sonis ST, Lindquist L, Van Vugt A, Stewart AA, Stam K, Qu GY, et al. Prevention of chemotherapy-induced ulcerative mucositis by transforming growth factor beta 3. Cancer Res (1994) 54(5):1135-8.

195. El-Habit OH, Saada HN, Azab KS, Abdel-Rahman M, El-Malah DF. The modifying effect of beta-carotene on gamma radiation-induced elevation of oxidative reactions and genotoxicity in male rats. Mutat Res (2000) 466(2):179-86. doi:10.1016/S1383-5718(00)00010-3

196. KonopackaM,WidelM,Rzeszowska-WolnyJ. Modifyingeffectofvitamins C,E and beta-carotene against gamma-ray-induced DNA damage in mouse cells. Mutat Res (1998) 417(2-3):85-94. doi:10.1016/S1383-5718(98)00095-3

197. Mills EE. The modifying effect of beta-carotene on radiation and chemotherapy induced oral mucositis. Br J Cancer (1988) 57(4):416-7. doi:10.1038/ bjc. 1988.94

198. Alfieri S, Ripamonti CI, Marceglia S, Orlandi E, Iacovelli NA, Granata R, et al. Temporal course and predictive factors of analgesic opioid requirement for chemoradiation-induced oral mucositis in oropharyngeal cancer. Head Neck (2016) 38(Suppl 1):E1521-7. doi:10.1002/hed.24272

199. Osaki T, Ueta E, Yoneda K, Hirota J, Yamamoto T. Prophylaxis of oral mucositis associated with chemoradiotherapy for oral carcinoma by azelastine hydrochloride (azelastine) with other antioxidants. Head Neck (1994) 16(4):331-9. doi:10.1002/hed.2880160407

200. Sato A, Saisho-Hattori T, Koizumi Y, Minegishi M, Iinuma K, Imaizumi M. Prophylaxis of mucosal toxicity by oral propantheline and cryotherapy in children with malignancies undergoing myeloablative chemo-radiotherapy. Tohoku J Exp Med (2006) 210(4):315-20. doi:10.1620/tjem.210.315

201. Mose S, Adamietz IA, Saran F, Thilmann C, Heyd R, Knecht R, et al. Can prophylactic application of immunoglobulin decrease 
radiotherapy-induced oral mucositis? Am J Clin Oncol (1997) 20(4):407-11. doi:10.1097/00000421-199708000-00018

202. Leborgne JH, Leborgne F, Zubizarreta E, Ortega B, Mezzera J. Corticosteroids and radiation mucositis in head and neck cancer. A double-blind placebo-controlled randomized trial. Radiother Oncol (1998) 47(2):145-8. doi:10.1016/S0167-8140(97)00174-6

203. Gruber S, Schmidt M, Bozsaky E, Wolfram K, Haagen J, Habelt B, et al. Modulation of radiation-induced oral mucositis by pentoxifylline: preclinical studies. Strahlenther Onkol (2015) 191(3):242-7. doi:10.1007/ s00066-014-0775-1

204. Verdi CJ, Garewal HS, Koenig LM, Vaughn B, Burkhead T. A double-blind, randomized, placebo-controlled, crossover trial of pentoxifylline for the prevention of chemotherapy-induced oral mucositis. Oral Surg Oral Med Oral Pathol Oral Radiol Endod (1995) 80(1):36-42. doi:10.1016/ S1079-2104(95)80014-X

205. Ferrà $\mathrm{C}$, de Sanjosé $\mathrm{S}$, Lastra $\mathrm{CF}$, Martí $\mathrm{F}$, Mariño EL, Sureda A, et al. Pentoxifylline, ciprofloxacin and prednisone failed to prevent transplantrelated toxicities in bone marrow transplant recipients and were associated with an increased incidence of infectious complications. Bone Marrow Transplant (1997) 20(12):1075-80. doi:10.1038/sj.bmt.1701023

206. Lopez-Jimenez J, Cancelas JA, Valino JM, Garcia-Larana J, Garcia-Avello A, Arranz MI, et al. Effects of oral pentoxifylline on TNF-alpha levels, transplant-related toxicities and engraftment after bone marrow transplantation. Bone Marrow Transplant (1994) 14(6):1011-2.

207. Stockschläder M, Kalhs P, Peters S, Zeller W, Krüger W, Kabisch H, et al. Intravenous pentoxifylline failed to prevent transplant-related toxicities in allogeneic bone marrow transplant recipients. Bone Marrow Transplant (1993) 12(4):357-62.

208. Kalhs P, Lechner K, Stockschlader M, Kruger W, Peters S, Zander A. Pentoxifylline did not prevent transplant-related toxicity in 31 consecutive allogeneic bone marrow transplant recipients. Blood (1992) 80(10):2683-4.

209. Sathishkumar S, Boyanovsky B, Karakashian AA, Rozenova K, Giltiay NV, Kudrimoti $\mathrm{M}$, et al. Elevated sphingomyelinase activity and ceramide concentration in serum of patients undergoing high dose spatially fractionated radiation treatment: implications for endothelial apoptosis. Cancer Biol Ther (2005) 4(9):979-86. doi:10.4161/cbt.4.9.1915

210. Jaffrézou JP, Bruno AP, Moisand A, Levade T, Laurent G. Activation of a nuclear sphingomyelinase in radiation-induced apoptosis. FASEB J (2001) 15(1):123-33. doi:10.1096/f.00-0305com

211. Pena LA, Fuks Z, Kolesnick RN. Radiation-induced apoptosis of endothelial cells in the murine central nervous system: protection by fibroblast growth factor and sphingomyelinase deficiency. Cancer Res (2000) 60(2):321-7.

212. Miranda SR, Erlich S, Visser JW, Gatt S, Dagan A, Friedrich VL Jr, et al. Bone marrow transplantation in acid sphingomyelinase-deficient mice: engraftment and cell migration into the brain as a function of radiation, age, and phenotype. Blood (1997) 90(1):444-52.

213. Chmura SJ, Mauceri HJ, Advani S, Heimann R, Beckett MA, Nodzenski E, et al. Decreasing the apoptotic threshold of tumor cells through protein kinase $\mathrm{C}$ inhibition and sphingomyelinase activation increases tumor killing by ionizing radiation. Cancer Res (1997) 57(19):4340-7.

214. SantanaP,PeñaLA, Haimovitz-Friedman A, MartinS, GreenD, McLoughlin M, et al. Acid sphingomyelinase-deficient human lymphoblasts and mice are defective in radiation-induced apoptosis. Cell (1996) 86(2):189-99. doi:10.1016/S0092-8674(00)80091-4

215. Levade T, Salvayre R, Potier M, Douste-Blazy L. Interindividual heterogeneity of molecular weight of human brain neutral sphingomyelinase determined by radiation inactivation method. Neurochem Res (1986) 11(8):1131-8. doi:10.1007/BF00965942

216. Levade T, Potier M, Salvayre R, Douste-Blazy L. Molecular weight of human brain neutral sphingomyelinase determined in situ by the radiation inactivation method. J Neurochem (1985) 45(2):630-2. doi:10.1111/j.1471-4159.1985. tb04033.x

217. Cruz Éde P, Campos L, Pereira Fda S, Magliano GC, Benites BM, Arana-Chavez VE, et al. Clinical, biochemical and histological study of the effect of antimicrobial photodynamic therapy on oral mucositis induced by 5 -fluorouracil in hamsters. Photodiagnosis Photodyn Ther (2015) 12(2): 298-309. doi:10.1016/j.pdpdt.2014.12.007
218. Donnelly JP, Bellm LA, Epstein JB, Sonis ST, Symonds RP. Antimicrobial therapy to prevent or treat oral mucositis. Lancet Infect Dis (2003) 3(7):405-12. doi:10.1016/S1473-3099(03)00668-6

219. Loury D, Embree JR, Steinberg DA, Sonis ST, Fiddes JC. Effect of local application of the antimicrobial peptide IB-367 on the incidence and severity of oral mucositis in hamsters. Oral Surg Oral Med Oral Pathol Oral Radiol Endod (1999) 87(5):544-51. doi:10.1016/S1079-2104(99)70131-9

220. Bondi E, Baroni C, Prete A, Gatti M, Carrassi A, Lodi G, et al. Local antimicrobial therapy of oral mucositis in paediatric patients undergoing bone marrow transplantation. Oral Oncol (1997) 33(5):322-6. doi:10.1016/ S1368-8375(97)00039-0

221. Kuroda K, Caputo GA. Antimicrobial polymers as synthetic mimics of host-defense peptides. Wiley Interdiscip Rev Nanomed Nanobiotechnol (2013) 5(1):49-66. doi:10.1002/wnan.1199

222. Takahashi H, Palermo EF, Yasuhara K, Caputo GA, Kuroda K. Molecular design, structures, and activity of antimicrobial peptide-mimetic polymers. Macromol Biosci (2013) 13(10):1285-99. doi:10.1002/mabi. 201300126

223. Scott RW, Tew GN. Mimics of host defense proteins; strategies for translation to therapeutic applications. Curr Top Med Chem (2017) 17(5):576-89.

224. Nicolatou-Galitis O, Velegraki A, Sotiropoulou-Lontou A, Dardoufas K, Kouloulias V, Kyprianou K, et al. Effect of fluconazole antifungal prophylaxis on oral mucositis in head and neck cancer patients receiving radiotherapy. Support Care Cancer (2006) 14(1):44-51. doi:10.1007/s00520005-0835-2

225. Lefebvre JL, Domenge C; Study Group of Mucositis. A comparative study of the efficacy and safety of fluconazole oral suspension and amphotericin B oral suspension in cancer patients with mucositis. Oral Oncol (2002) 38(4):337-42. doi:10.1016/S1368-8375(01)00063-X

226. Shenep JL. Combination and single-agent empirical antibacterial therapy for febrile cancer patients with neutropenia and mucositis. NCI Monogr (1990) 9:117-22.

227. SpijkervetFK, Van Saene HK, Van Saene JJ, Panders AK, Vermey A, Mehta DM, et al. Effect of selective elimination of the oral flora on mucositis in irradiated head and neck cancer patients. J Surg Oncol (1991) 46(3):167-73. doi:10.1002/jso.2930460309

228. Matthews RH, Ercal N. Prevention of mucositis in irradiated head and neck cancer patients. J Exp Ther Oncol (1996) 1(2):135-8.

229. Spijkervet FK, van Saene HK, van Saene JJ, Panders AK, Vermey A, Mehta DM. Mucositis prevention by selective elimination of oral flora in irradiated head and neck cancer patients. J Oral Pathol Med (1990) 19(10):486-9. doi:10.1111/ j.1600-0714.1990.tb00792.x

230. Saral R, Burns WH, Prentice HG. Herpes virus infections: clinical manifestations and therapeutic strategies in immunocompromised patients. Clin Haematol (1984) 13(3):645-60.

231. Prelack MS, Patterson KR, Berger JR. Varicella zoster virus rhombencephalomyelitis following radiation therapy for oropharyngeal carcinoma. J Clin Neurosci (2016) 25:164-6. doi:10.1016/j.jocn.2015.09.009

232. Vaughan G, Rodríguez-Castillo A, Cruz-Rivera MY, Ruiz-Tovar K, RamírezGonzález JE, Rivera-Osorio P, et al. Is ultra-violet radiation the main force shaping molecular evolution of varicella-zoster virus? Virol J (2011) 8:370. doi:10.1186/1743-422X-8-370

233. Evans TG, Bernstein DI, Raborn GW, Harmenberg J, Kowalski J, Spruance SL. Double-blind, randomized, placebo-controlled study of topical 5\% acyclovir-1\% hydrocortisone cream (ME-609) for treatment of UV radiationinduced herpes labialis. Antimicrob Agents Chemother (2002) 46(6):1870-4. doi:10.1128/AAC.46.6.1870-1874.2002

234. Jagetia GC, Aruna R, Nayak BS. Alteration in the radiation-induced LD release in HeLa cells by acyclovir. Clin Chim Acta (2000) 294(1-2):129-38. doi:10.1016/S0009-8981(00)00180-7

235. Jagetia GC, Aruna R. Effect of acyclovir on the radiation-induced micronuclei and cell death. Mutat Res (2000) 469(1):9-21. doi:10.1016/ S1383-5718(00)00048-6

236. Bubley GJ, Chapman B, Chapman SK, Crumpacker CS, Schnipper LE. Effect of acyclovir on radiation- and chemotherapy-induced mouth lesions. Antimicrob Agents Chemother (1989) 33(6):862-5. doi:10.1128/ AAC. 33.6.862 
237. Spruance SL. Cutaneous herpes simplex virus lesions induced by ultraviolet radiation. A review of model systems and prophylactic therapy with oral acyclovir. Am J Med (1988) 85(2A):43-5.

238. Sougawa M, Akagi K, Murata T, Kawasaki S, Sawada S, Yoshii G, et al. Enhancement of radiation effects by acyclovir. Int J Radiat Oncol Biol Phys (1986) 12(8):1537-40. doi:10.1016/0360-3016(86)90211-7

239. Schmidt M, Haagen J, Noack R, Siegemund A, Gabriel P, Dörr W. Effects of bone marrow or mesenchymal stem cell transplantation on oral mucositis (mouse) induced by fractionated irradiation. Strahlenther Onkol (2014) 190(4):399-404. doi:10.1007/s00066-013-0510-3

240. Schmidt M, Piro-Hussong A, Siegemund A, Gabriel P, Dörr W. Modification of radiation-induced oral mucositis (mouse) by adult stem cell therapy: single-dose irradiation. Radiat Environ Biophys (2014) 53(4):629-34. doi:10.1007/s00411-014-0552-7

241. Shieh SH, Wang ST, Tsai ST, Tseng CC. Mouth care for nasopharyngeal cancer patients undergoing radiotherapy. Oral Oncol (1997) 33(1):36-41.

242. Rugg T, Saunders MI, Dische S. Smoking and mucosal reactions to radiotherapy. Br J Radiol (1990) 63(751):554-6. doi:10.1259/0007-1285-63-751-554

243. Scherlacher A, Beaufort-Spontin F. [Radiotherapy of head-neck neoplasms: prevention of inflammation of the mucosa by sucralfate treatment]. HNO (1990) 38(1):24-8.

244. Carter DL, Hebert ME, Smink K, Leopold KA, Clough RL, Brizel DM. Double blind randomized trial of sucralfate vs placebo during radical radiotherapy for head and neck cancers. Head Neck (1999) 21(8):760-6.

245. Feber T. Management of mucositis in oral irradiation. Clin Oncol ( $R$ Coll Radiol) (1996) 8(2):106-11.

246. Spijkervet FK, van Saene HK, Panders AK, Vermey A, van Saene JJ, Mehta $\mathrm{DM}$, et al. Effect of chlorhexidine rinsing on the oropharyngeal ecology in patients with head and neck cancer who have irradiation mucositis. Oral Surg Oral Med Oral Pathol (1989) 67(2):154-61.

247. Ferretti GA, Raybould TP, Brown AT, Macdonald JS, Greenwood M, Maruyama Y, et al. Chlorhexidine prophylaxis for chemotherapy- and radiotherapy-induced stomatitis: a randomized double-blind trial. Oral Surg Oral Med Oral Pathol (1990) 69(3):331-8.

248. Hasenau C, Clasen BP, Roettger D. [Use of standardized oral hygiene in the prevention and therapy of mucositis in patients treated with radiochemotherapy of head and neck neoplasms]. Laryngol Rhinol Otol (Stuttg) (1988) 67(11):576-9.

249. Symonds RP, McIlroy P, Khorrami J, Paul J, Pyper E, Alcock SR, et al. The reduction of radiation mucositis by selective decontamination antibiotic pastilles: a placebo-controlled double-blind trial. Br J Cancer (1996) 74(2):312-7.

250. Okuno SH, Foote RL, Loprinzi CL, Gulavita S, Sloan JA, Earle J, et al. A randomized trial of a nonabsorbable antibiotic lozenge given to alleviate radiation-induced mucositis. Cancer (1997) 79(11):2193-9.

251. Carl W, Emrich LS. Management of oral mucositis during local radiation and systemic chemotherapy: a study of 98 patients. J Prosthet Dent (1991) 66(3):361-9.

252. Abdelaal AS, Barker DS, Fergusson MM. Treatment for irradiation-induced mucositis. Lancet (1989) 1(8629):97.

253. Kim JH, Chu FC, Lakshmi V, Houde R. Benzydamine HCl, a new agent for the treatment of radiation mucositis of the oropharynx. Am J Clin Oncol (1986) 9(2):132-4.

254. Samaranayake LP, Robertson AG, MacFarlane TW, Hunter IP, MacFarlane G, Soutar DS, et al. The effect of chlorhexidine and benzydamine mouthwashes on mucositis induced by therapeutic irradiation. Clin Radiol (1988) 39(3):291-4.

255. Prada A, Chiesa F. Effects of benzydamine on the oral mucositis during antineoplastic radiotherapy and/or intra-arterial chemotherapy. Int J Tissue React (1987) 9(2):115-9.
256. Porteder H, Rausch E, Kment G, Watzek G, Matejka M, Sinzinger H. Local prostaglandin E2 in patients with oral malignancies undergoing chemo- and radiotherapy. J Craniomaxillofac Surg (1988) 16(8):371-4.

257. Maciejewski B, Zajusz A, Pilecki B, Swiatnicka J, Skladowski K, Dorr $\mathrm{W}$, et al. Acute mucositis in the stimulated oral mucosa of patients during radiotherapy for head and neck cancer. Radiother Oncol (1991) 22(1):7-11.

258. Bourhis J, De Crevoisier R, Abdulkarim B, Deutsch E, Lusinchi A, Luboinski $\mathrm{B}$, et al. A randomized study of very accelerated radiotherapy with and without amifostine in head and neck squamous cell carcinoma. Int J Radiat Oncol Biol Phys (2000) 46(5):1105-8.

259. Koukourakis MI, Kyrias G, Kakolyris S, Kouroussis C, Frangiadaki C, Giatromanolaki A, et al. Subcutaneous administration of amifostine during fractionated radiotherapy: a randomized phase II study. J Clin Oncol (2000) 18(11):2226-33. doi:10.1200/JCO.2000.18.11.2226

260. Schonekas KG, Wagner W, Prott FJ. Amifostine-a radioprotector in locally advanced head and neck tumors. Strahlenther Onkol (1999) 175(Suppl 4):27-9.

261. Wagner W, Prott FJ, Schonekas KG. Amifostine: a radioprotector in locally advanced head and neck tumors. Oncol Rep (1998) 5(5):1255-7.

262. Buntzel J, Kuttner K, Frohlich D, Glatzel M. Selective cytoprotection with amifostine in concurrent radiochemotherapy for head and neck cancer. Ann Oncol (1998) 9(5):505-9.

263. Peters K, Mucke R, Hamann D, Ziegler PG, Fietkau R. Supportive use of amifostine in patients with head and neck tumors undergoing radio-chemotherapy. Is it possible to limit the duration of the application of amifostine? Strahlenther Onkol (1999) 175(Suppl 4):23-6.

264. Vacha P, Marx M, Engel A, Richter E, Feyerabend T. [Side effects of postoperative radiochemotherapy with amifostine versus radiochemotherapy alone in head and neck tumors. Preliminary results of a prospective randomized trial]. Strahlenther Onkol (1999) 175(Suppl 4):18-22.

265. Wagner W, Alfrink M, Haus U, Matt J. Treatment of irradiation-induced mucositis with growth factors (rhGM-CSF) in patients with head and neck cancer. Anticancer Res (1999) 19(1B):799-803.

266. Rosso M, Blasi G, Gherlone E, Rosso R. Effect of granulocyte-macrophage colony-stimulating factor on prevention of mucositis in head and neck cancer patients treated with chemo-radiotherapy. J Chemother (1997) 9(5):382-5. doi:10.1179/joc.1997.9.5.382

267. Mascarin M, Franchin G, Minatel E, Gobitti C, Talamini R, De Maria D, et al. The effect of granulocyte colony-stimulating factor on oral mucositis in head and neck cancer patients treated with hyperfractionated radiotherapy. Oral Oncol (1999) 35(2):203-8.

268. Schneider SB, Nishimura RD, Zimmerman RP, Tran L, Shiplacoff J, Tormey $\mathrm{M}$, et al. Filgrastim (r-metHuG-CSF) and its potential use in the reduction of radiation-induced oropharyngeal mucositis: an interim look at a randomized, double-blind, placebo-controlled trial. Cytokines Cell Mol Ther (1999) 5(3):175-80.

Conflict of Interest Statement: The authors declare that the research was conducted in the absence of any commercial or financial relationships that could be construed as a potential conflict of interest.

Copyright (C) 2017 Maria, Eliopoulos and Muanza. This is an open-access article distributed under the terms of the Creative Commons Attribution License (CC BY). The use, distribution or reproduction in other forums is permitted, provided the original author(s) or licensor are credited and that the original publication in this journal is cited, in accordance with accepted academic practice. No use, distribution or reproduction is permitted which does not comply with these terms. 